Cite this: Phys. Chem. Chem. Phys., 2011, 13, 19378-19392

\title{
Structure and dynamics of the fast lithium ion conductor " $\mathrm{Li}_{7} \mathrm{La}_{3} \mathrm{Zr}_{2} \mathrm{O}_{12}$ " $\dagger$
}

\author{
Henrik Buschmann, ${ }^{a}$ Janis Dölle, ${ }^{a}$ Stefan Berendts, ${ }^{a}$ Alexander Kuhn, ${ }^{b}$ \\ Patrick Bottke, ${ }^{b}$ Martin Wilkening, ${ }^{b}$ Paul Heitjans, ${ }^{b}$ Anatoliy Senyshyn, ${ }^{c}$ \\ Helmut Ehrenberg, ${ }^{d}$ Andriy Lotnyk, ${ }^{e}$ Viola Duppel, ${ }^{f}{ }_{\text {Lorenz Kienle }}^{e}$ and \\ Jürgen Janek*a
}

Received 28th June 2011, Accepted 30th August 2011

DOI: $10.1039 / \mathrm{c} 1 \mathrm{cp22108f}$

The solid lithium-ion electrolyte " $\mathrm{Li}_{7} \mathrm{La}_{3} \mathrm{Zr}_{2} \mathrm{O}_{12}$ " (LLZO) with a garnet-type structure has been prepared in the cubic and tetragonal modification following conventional ceramic syntheses routes. Without aluminium doping tetragonal LLZO was obtained, which shows a two orders of magnitude lower room temperature conductivity than the cubic modification. Small concentrations of $\mathrm{Al}$ in the order of $1 \mathrm{wt} \%$ were sufficient to stabilize the cubic phase, which is known as a fast lithium-ion conductor. The structure and ion dynamics of Al-doped cubic LLZO were studied by impedance spectroscopy, dc conductivity measurements, ${ }^{6} \mathrm{Li}$ and ${ }^{7} \mathrm{Li} \mathrm{NMR}, \mathrm{XRD}$, neutron powder diffraction, and TEM precession electron diffraction. From the results we conclude that aluminium is incorporated in the garnet lattice on the tetrahedral $24 d \mathrm{Li}$ site, thus stabilizing the cubic LLZO modification. Simulations based on diffraction data show that even at the low temperature of $4 \mathrm{~K}$ the $\mathrm{Li}$ ions are blurred over various crystallographic sites. This strong $\mathrm{Li}$ ion disorder in cubic Al-stabilized LLZO contributes to the high conductivity observed. The Li jump rates and the activation energy probed by NMR are in very good agreement with the transport parameters obtained from electrical conductivity measurements. The activation energy $E_{\mathrm{a}}$ characterizing longrange ion transport in the $\mathrm{Al}$-stabilized cubic LLZO amounts to $0.34 \mathrm{eV}$. Total electric conductivities determined by ac impedance and a four point de technique also agree very well and range from $1 \times$ $10^{-4} \mathrm{Scm}^{-1}$ to $4 \times 10^{-4} \mathrm{Scm}^{-1}$ depending on the $\mathrm{Al}$ content of the samples. The room temperature conductivity of Al-free tetragonal LLZO is about two orders of magnitude lower $\left(2 \times 10^{-6} \mathrm{Scm}^{-1}\right.$, $E_{\text {a }}=0.49 \mathrm{eV}$ activation energy). The electronic partial conductivity of cubic LLZO was measured using the Hebb-Wagner polarization technique. The electronic transference number $t_{\mathrm{e}-}$ is of the order of $10^{-7}$. Thus, cubic LLZO is an almost exclusive lithium ion conductor at ambient temperature.

${ }^{a}$ Physikalisch-Chemisches Institut, Justus Liebig Universität Giessen Heinrich-Buff-Ring 58, 35392 Gießen, Germany.

E-mail: juergen.janek@phys.chemie.uni-giessen.de

${ }^{b}$ Institut für Physikalische Chemie und Elektrochemie,

Leibniz Universität Hannover, Callinstraße $3 a$,

30167 Hannover, Germany

${ }^{c}$ Forschungsneutronenquelle Heinz Maier-Leibnitz (FRM II),

Technische Universität München, Lichtenbergstrasse 1,

85747 Garching, Germany

dInstitute for Applied Materials (IAM), Karlsruhe Institute of

Technology (KIT), Hermann-von-Helmholtz-Platz 1,

76344 Eggenstein-Leopoldshafen, Germany

${ }^{e}$ Faculty of Engineering, Institute for Material Science,

Synthesis and Real Structure, Christian Albrechts Universität zu Kiel,

Kaiserstr. 2, D-24143 Kiel, Germany

${ }^{f}$ Max Planck Institute for Solid State Research, Heisenbergstr. 1,

D-70569 Stuttgart, Germany

$\dagger$ Electronic supplementary information (ESI) available: TEM pictures.

CCDC reference numbers 833727 . For ESI and crystallographic data in

CIF or other electronic format see DOI: $10.1039 / \mathrm{clcp} 22108 \mathrm{f}$

\section{Introduction}

Today, state-of-the-art electrolytes in lithium (ion) batteries are composed of organic solvents or polymers with a dissolved Li-salt. ${ }^{1}$ These are combustible in case of abuse, and they often react with electrode materials to form passivating films in the best case. ${ }^{2,3}$ This is on the one hand necessary and favourable, on the other hand proceeding film growth and electrolyte decomposition at elevated temperatures lead to degradation of the battery and shorter battery life times. ${ }^{4}$ In particular, new cell concepts based on high voltage cathodes or mobile non-metal cathode systems based on sulfur or oxygen require improved electrolytes, and it may well be that ion-conducting membranes become indispensable. Therefore, the search for stable and incombustible inorganic solid electrolytes with high lithium ion conductivity is currently an important issue in battery research. 
Recently $\mathrm{Li}$ ion conducting garnet-type oxides are considered as promising electrolytes because of their high conductivity and stability $v s$. Li metal. Thangadurai et al. first reported about $\mathrm{Li}$ ion mobility in $\mathrm{Li}_{5} \mathrm{La}_{3} M_{2} \mathrm{O}_{12}(M=\mathrm{Nb}$, Ta). ${ }^{5}$ After the report on cubic $\mathrm{Li}_{7} \mathrm{La}_{3} \mathrm{Zr}_{2} \mathrm{O}_{12}$ (LLZO) with the so far highest room temperature conductivity ${ }^{6}$ in 2007 these materials have attracted a lot of interest, but it was often found to be difficult to obtain highly conductive LLZO. Later Awaka et al. showed that the reason for this is the existence of a second, tetragonally distorted modification ${ }^{7}$ with a much lower conductivity than cubic LLZO. Although some groups reported about the successful preparation of cubic LLZO it is impossible to judge about the quality of the reported materials, due to missing experimental data like conductivities and/or X-ray diffractograms (XRD). ${ }^{8,9}$ Recently, some groups reported on aluminium impurities and the formation of cubic LLZO in combination with $\mathrm{Al}$ impurities or Al doping. ${ }^{10-13}$ None of these groups could clarify the exact effect of $\mathrm{Al}$ on the formation of cubic LLZO and the changes of the crystal structure caused by $\mathrm{Al}$ incorporation except the suggestion that $\mathrm{Al}$ might substitute $\mathrm{Li}$ in the garnet lattice. In order to indicate that it is in fact not pure $\mathrm{Li}_{7} \mathrm{La}_{3} \mathrm{Zr}_{2} \mathrm{O}_{12}$ which shows high Li ion conductivity, we will use the abbreviation LLZO or the formula in quotation marks throughout the paper.

Despite attempts to clarify the crystal structure of cubic LLZO by single crystal $\mathrm{XRD}^{8,10}$ not much is known about the lithium distribution in cubic LLZO, owing to the small scattering factors of lithium ions. It is the aim of this study to gain deeper insight into the structure and ion dynamics of cubic LLZO in order to better judge its potential as a battery electrolyte. In particular, we made a systematic study of the effect of aluminium doping on the formation of the tetragonal and the cubic LLZO modification. In order to obtain maximal structural information we combined XRD, neutron powder diffraction as well as transmission electron microscopy/precession electron diffraction (TEM-PED) and nuclear magnetic resonance (NMR) spectroscopy. We propose a structure model which takes the lithium ions properly into account as well as the aluminium dopant. In order to also obtain comprehensive information on the lithium ion dynamics we combined electrochemical measurements and ${ }^{7} \mathrm{Li}$ NMR spin-spin as well as spin-lattice relaxation techniques. Very good agreement of the different results was found. Finally, we used the Hebb-Wagner method to determine the electronic transference number of cubic LLZO which has not been reported before so far.

\section{Experimental}

\section{Synthesis}

LLZO was prepared by a high temperature route using $\mathrm{LiOH}$ (Chempur, 99\%, anhydrous), $\mathrm{ZrO}_{2}$ (Chempur, 99.9\%) and $\mathrm{La}_{2} \mathrm{O}_{3}$ (Chempur $99.99 \%$, dried at $900{ }^{\circ} \mathrm{C}$ for $12 \mathrm{~h}$ ). The starting materials were mixed in stoichiometric amounts (10 wt $\%-20 \mathrm{wt} \% \mathrm{LiOH}$ excess was used) and milled for $8 \mathrm{~h}-12 \mathrm{~h}$ in 2-propanol in a planetary ball mill (Fritsch, Pulverisette 7 premium line or Fritsch, Pulverisette 5 classic line) with zirconia balls and grinding bowls. We made use of three different procedures to prepare Al-free and Al-containing LLZO samples. Interestingly, although we followed the preparation route described in the literature ${ }^{6}$ to synthesize cubic LLZO, the second calcination step at $1130{ }^{\circ} \mathrm{C}$ led to the formation of the tetragonal modification ${ }^{7}$ of LLZO. The successful preparation of cubic LLZO turned out to depend on the number of calcination steps and the kind of crucibles used. The cubic phase of LLZO only formed when alumina crucibles were used and when the powder was annealed several times at $1130{ }^{\circ} \mathrm{C}$ for about $12 \mathrm{~h}$ with milling steps in between. As a result of the reaction between the crucible and the powder a thinning of the crucible after some preparation steps was observed. Several batches of cubic LLZO were prepared according to this procedure and investigated by ICP-OES (inductively coupled plasma optical emission spectroscopy). All of the cubic LLZO samples prepared showed an Al content of about $0.9 \mathrm{wt} \%$ ( $28 \mathrm{~mol} \% \mathrm{Al}$ per $\left.\mathrm{mol} \mathrm{Li}_{7} \mathrm{La}_{3} \mathrm{Zr}_{2} \mathrm{O}_{12}\right)$. To verify the hypothesis that $\mathrm{Al}$ stabilizes the cubic modification we prepared LLZO samples intentionally doped with $\mathrm{Al}$ as well as free of $\mathrm{Al}$. For this purpose crucibles free of any $\mathrm{Al}$ traces have been used. A small amount (corresponding to 0.5 $\mathrm{wt} \%$ and $0.9 \mathrm{wt} \%$ in LLZO) of $\gamma-\mathrm{Al}_{2} \mathrm{O}_{3}$ (Merck, anhydrous) was added to the mixture of the starting materials. The Al-doped samples were otherwise prepared according to the procedure described above. After calcination at $900{ }^{\circ} \mathrm{C}$ and $1130{ }^{\circ} \mathrm{C}$ the powder was pressed to pellets, covered with mother powder, and sintered at $1230{ }^{\circ} \mathrm{C}$ for $30 \mathrm{~h}$. For comparison, Al-free LLZO has been prepared without addition of $\gamma-\mathrm{Al}_{2} \mathrm{O}_{3}$ powder.

\section{Phase characterization}

Phase analysis along synthesis was carried out on a Siemens D500 Siemens AG, now Bruker AXS in Bragg-Bretano geometry with $\mathrm{CuK} \alpha$-radiation. Phase analysis and determination of cell parameters at room temperature in cubic LLZO were carried out using X-ray powder diffraction (XPD) with a STOE STADI $\mathrm{P}$ diffractometer either by using $\mathrm{MoK} \alpha_{1}$ radiation, $\lambda=0.7093 \AA$ or $\mathrm{CoK} \alpha_{1}$-radiation, $\lambda=1.78897 \AA$.

Elastic coherent neutron scattering experiments were performed on the high-resolution powder diffractometer SPODI at the research reactor FRM-II (Garching, Germany). ${ }^{14}$ Monochromatic neutrons $(\lambda=1.5482 \AA)$ were obtained at a $155^{\circ}$ take-off angle using the (551) reflection of a verticallyfocused composite Ge monochromator. The vertical positionsensitive multidetector ( $300 \mathrm{~mm}$ effective height) consisting of $80{ }^{3} \mathrm{He}$ tubes and covering an angular range of $160^{\circ} .2 \theta$ was used for data collection. Measurements were performed in Debye-Scherrer geometry. The powder sample was filled into a thin-wall $(0.15 \mathrm{~mm})$ vanadium can of $13 \mathrm{~mm}$ in diameter and then mounted in the top-loading closed-cycle refrigerator. Helium 4 was used as a heat transmitter. The instantaneous temperature was measured using two thin film resistance cryogenic temperature sensors Cernox and controlled by a temperature controller from LakeShore. Two-dimensional powder diffraction data were collected at $4 \mathrm{~K}$ and $300 \mathrm{~K}$ using two wavelengths and then corrected for geometrical aberations and curvature of Debye-Scherrer rings. 
The analysis of powder diffraction data was carried out using the Rietveld technique implemented into the software package FullProf. ${ }^{15}$ The peak profile was described by a modified pseudo-Voigt function (Thompson-Cox-Hastings). The background of the diffraction patterns was fitted using a linear interpolation between selected data points in nonoverlapping regions. Cylindrical absorption correction with $\mu R=0.7072$ has been applied to obtain neutron intensities. The scale factor, lattice parameter, fractional coordinates of atoms and their iso-/anisotropic displacement parameters, zero angular shift, profile shape parameters and half width (Caglioti) parameters were varied during the fitting. Prior to the Rietveld refinement route the lattice parameters, background and profile shape parameters were estimated using the full profile decomposition (Le-Bail) technique.

The maximum entropy method (MEM) implemented in the program PRIMA ${ }^{16}$ was used to determine the nuclear densities. MEM deals with the 3D densities in the way giving the maximum variance of structure factors $F_{\mathrm{c}}(\mathrm{MEM})$ within standard deviation of observed structure factors $F_{\text {o, }}$, where structural information can effectively be extracted from the diffraction data and reflected on the resulting threedimensional distribution of electron/nuclear densities. MEM analysis was conducted using either 189 (at $4 \mathrm{~K}$ ) or 192 (at $300 \mathrm{~K}$ ) structure factors obtained from the Rietveld analysis, with the unit cell divided into $128 \times 128 \times 128$ pixels.

\section{TEM investigations}

Transmission electron microscopy (TEM) was performed in a Philips CM 30 STwin microscope $(\mathrm{Cs}=1.15 \mathrm{~mm}, 300 \mathrm{kV}$, $\mathrm{LaB}_{6}$ ) equipped with a Spinning Star precession module (Nanomegas) and an EDX detector (Fa. Noran). Energy dispersive X-ray (EDX) analyses were performed in a Tecnai F30 STwin microscope $(\mathrm{Cs}=1.2 \mathrm{~mm}, 300 \mathrm{kV}$, FEG) equipped with a $\mathrm{Si} / \mathrm{Li}$ detector (EDAX system). The analyses were carried out in scanning transmission electron microscope (STEM) mode using the HAADF detector for image acquisition. The samples for TEM investigations were grinded and suspended in n-butanol. One drop of each suspension was placed on a TEM support (a lacey carbon film on a copper grid). All images were recorded with a Gatan Multiscan CCD camera $(1 \mathrm{k} \times 1 \mathrm{k})$ and evaluated with a program Digital Micrograph (Gatan, Inc.). Identification of the crystal modification of LLZO was performed by comparing the diffracted intensities of simulated and experimental precession electron diffraction (PED) images. The precession angle was set to $3^{\circ}$ for the PED experiments. The selected area electron diffraction (SAED) and PED patterns were recorded along different zone axes. Several zone axes were examined, however, in the present work we are focusing on [001] since this direction represents the most significant one for phase distinction. The software $\mathrm{JEMS}^{17}$ was used for the simulations of SAED and PED patterns (including multiple scattering events (dynamical calculations)) as well as for simulations of high-resolution TEM (HRTEM) images (using the multislice approach). The Weickenmeier-Kohl atomic form factor was used for the simulations. For PED calculations the sample thickness was set to $10 \mathrm{~nm}$. For HRTEM simulations the following microscope parameters were used: Cs $=1.15 \mathrm{~mm}$, defocus spread $7 \mathrm{~nm}$ and illumination semiangle of $0.8 \mathrm{mrad}$. In order to reduce noise, all HRTEM images were filtered after Fast Fourier transformation using a band-pass mask, with no loss of essential structural information in the following. The structural data for PED simulations of tetragonal LLZO were taken from ref. 7.

\section{NMR measurements}

${ }^{7} \mathrm{Li}$ (spin-quantum number $I=3 / 2$ ) NMR spin-spin- and spin-lattice relaxation rates in both the laboratory and rotating frame of reference were recorded using a modified MSL 100 spectrometer and an MSL 400 spectrometer operating at $4.7 \mathrm{~T}(77.8 \mathrm{MHz})$ and $9.4 \mathrm{~T}(155.5 \mathrm{MHz})$, respectively. The spectrometers were connected to Oxford cryomagnets. Standard broadband probes (Bruker) as well as home-built high temperature probes were used for data acquisition. $90^{\circ}$ pulse lengths ranged from $4 \mu$ s to $6 \mu$ s. The temperature in the sample chamber was monitored with the help of an Oxford ITC4 using $\mathrm{Ni}-\mathrm{CrNi}$ thermocouples to within $\pm 2 \mathrm{~K}$. Temperatures below room temperature were adjusted with the help of a flow of freshly evaporated nitrogen. Above $290 \mathrm{~K}$ the temperature in the sample chamber was controlled with a stream of heated air. ${ }^{7} \mathrm{Li}$ NMR spin-lattice relaxation rates $\left(1 / T_{1}=R_{1}\right)$ in the laboratory frame of reference were recorded using a saturation recovery pulse sequence: a $90^{\circ}$ detection pulse is sent after a comb of closely spaced saturation pulses of the same duration. The corresponding transients $M_{z}(t)$ describing the recovery of longitudinal magnetization follow single exponential time behaviour. This is in contrast to the analogous measurements of $1 / T_{1 \rho}=R_{1 \rho}$ in the rotating frame of reference performed using the spin-lock technique at frequencies of $11.5 \mathrm{kHz}$ and $33.1 \mathrm{kHz}$. The transients $M_{(x y)^{\prime}}(t)$ can be well parameterized by stretched exponentials $M_{(x y)^{\prime}}(t) \propto \exp \left(-\left(t / T_{1 \rho}\right)^{\gamma}\right)$ with $0.4<\gamma<1$.

High-resolution ${ }^{6} \mathrm{Li}$ and ${ }^{27} \mathrm{Al}$ NMR spectra under fast sample rotation were recorded using an Avance III spectrometer (Bruker) in combination with a shimmed cryomagnet of $14.1 \mathrm{~T}$. Magic angle spinning (MAS) spectra were recorded with a $2.5-\mathrm{mm}$-probe at $88.4 \mathrm{MHz}$ and $156.5 \mathrm{MHz}$, respectively. The spinning speed was $30 \mathrm{kHz}$.

\section{Electrochemical characterization}

Pellets of sintered LLZO samples were cut into pieces of different lengths and vacuum dried at $100{ }^{\circ} \mathrm{C}$ for at least $6 \mathrm{~h}$. The dried pellets were then exclusively handled in an argon filled glove box (MBraun, Labmaster) with less than $0.1 \mathrm{ppm}$ $\mathrm{H}_{2} \mathrm{O}$ and $\mathrm{O}_{2}$ in the gas. Lithium foil (Chemetall) was used as electrode material for conductivity measurements by two electrode ac impedance spectroscopy and four electrode dc techniques. The lithium foil was pressed on the sides of the LLZO pellets (Fig. 1a) and then annealed at $170{ }^{\circ} \mathrm{C}$ for some minutes on a heating plate to improve the contact between $\mathrm{Li}$ and LLZO. For the dc measurements two additional electrodes were placed in the flank of the pellet. Therefore, two $0.5 \mathrm{~mm}$ wide grooves were cut in the flank and filled with $\mathrm{Li}$ metal (Fig. 1b). For both ac and dc measurements the Li 
a)

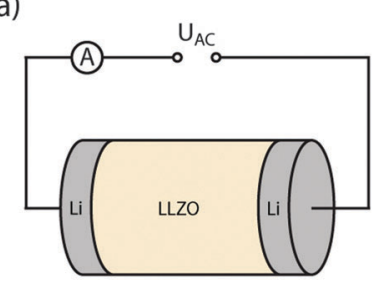

c)

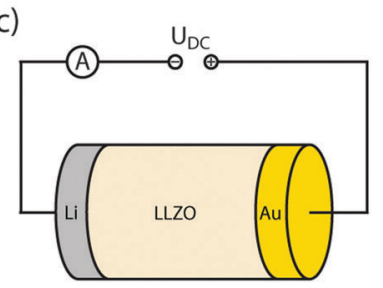

b)

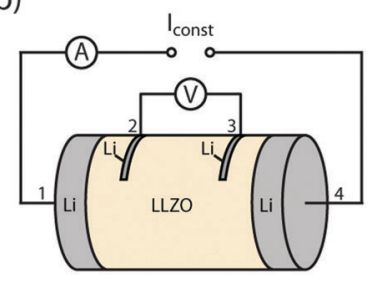

Fig. 1 Scheme of different electrode arrangements (a) for ac conductivity measurements with two lithium electrodes, (b) for dc conductivity measurements with four lithium electrodes and (c) for Hebb-Wagner type electronic conductivity measurements with one lithium and one $\mathrm{Li}$ ion blocking gold electrode.

electrodes were contacted with a silver or copper wire. These cells were sealed in gas-tight pouches of aluminized compound foil. Temperature dependent conductivity measurements were carried out in a temperature chamber (WTL 64 Weiss Technik) in the temperature range from $-40{ }^{\circ} \mathrm{C}$ to $100{ }^{\circ} \mathrm{C}$. A potentiostat/galvanostat (model SP-300 from Biologic Science Instruments) was employed for electrochemical characterization. The software package EC-Lab V10.02 was used for data acquisition and impedance data fitting. Impedance spectroscopy was carried out in the frequency range from $7 \mathrm{MHz}$ to $1 \mathrm{~Hz}$ (amplitude of $20 \mathrm{mV}$ ).

Additionally, low-temperature impedance spectra down to $-148{ }^{\circ} \mathrm{C}$ were recorded by employing a Novocontrol Concept 80 broadband dielectric spectrometer which is equipped with a BDS 1200 sample cell and a BETA analyzer. The latter is capable to measure impedances down to $10^{-14} \Omega$ at frequencies ranging from a few $\mu \mathrm{Hz}$ to $20 \mathrm{MHz}$. Temperature regulation and controlling within an accuracy of about $0.5 \mathrm{~K}$ was carried out with a Quattro cryosystem (Novocontrol) using dry nitrogen gas. The root mean square ac voltage was typically $0.1 \mathrm{~V}$ to $1.0 \mathrm{~V}$. These data were mainly used to extract electrical relaxation rates from the corresponding modulus spectra.

The dc conductivity was measured by applying a constant current in the range from $1 \mu \mathrm{A}-100 \mu \mathrm{A}$ between the electrodes 1 and 4 (Fig. 1b) and measuring the resulting voltage drop over the distance of the potential probes 2 and 3 (Fig. 1b). At each temperature a constant dc current was applied for $90 \mathrm{~s}$. The voltage drop was simultaneously measured and remained constant over the measured time period.

The electronic partial conductivity of cubic LLZO was measured using the Hebb-Wagner technique. ${ }^{18,19} \mathrm{~A}$ gold blocking electrode of approximately $1 \mu \mathrm{m}$ thickness was deposited on one of the pellets surfaces by thermal $\mathrm{Au}$ evaporation. As mentioned above, Li foil was attached on the opposing surface as a reversible electrode for $\mathrm{Li}$ ions (Fig. 1c). Measurements were performed in potentiostatic

mode (Keithley 6430) in a home-built cell in the range from $2.5 \mathrm{~V}$ and $4.5 \mathrm{~V} v s . \mathrm{Li} / \mathrm{Li}^{+}$in an argon filled glove box with the Li electrode connected as cathode. Steady-state was assumed when the current remained constant within $10 \%$ over a period of several hours.

\section{Results and discussion}

\section{Synthesis}

Synthesis of cubic LLZO was only successful when an aluminium source was present during synthesis. Thus cubic LLZO formed either by working in $\mathrm{Al}_{2} \mathrm{O}_{3}$ crucibles, where the LLZO starting materials react with the crucible, or when intentionally using $\gamma-\mathrm{Al}_{2} \mathrm{O}_{3}$ powder as a dopant in the LLZO synthesis. Preparation of cubic LLZO in $\mathrm{Al}_{2} \mathrm{O}_{3}$ crucibles without further doping takes more than two calcination and milling steps due to the stepwise reaction of the powder at the crucible walls only. When the formation of phase pure cubic LLZO (by reaction with the wall) was completed the aluminium content of these samples was typically about $0.9 \mathrm{wt} \%$ as determined by ICP-OES. The Al free synthesis did not lead to pure cubic LLZO but rather to the tetragonal modification or a mixture of the cubic and tetragonal modifications of LLZO (Fig. 2a-b). Synthesis with $0.5 \mathrm{wt} \% \mathrm{Al}_{2} \mathrm{O}_{3}$ led mainly to cubic LLZO, however, the corresponding reflexes are somewhat broader indicating that there might be some tetragonal LLZO present as well (Fig. 2c). Doping with $0.9 \mathrm{wt} \% \mathrm{Al}_{2} \mathrm{O}_{3}$ in $\mathrm{Al}$ free crucibles as well as synthesis without doping but using $\mathrm{Al}_{2} \mathrm{O}_{3}$ crucibles led to cubic LLZO (Fig. $2 \mathrm{~d}$ and e). These results clearly prove that the aluminium content plays a crucial role for the formation of phase pure cubic LLZO.

\section{Structural information from MAS NMR}

Structural features of Al-doped cubic LLZO were elucidated by ${ }^{27} \mathrm{Al}$ MAS NMR spectroscopy. In Fig. 3 the NMR spectra

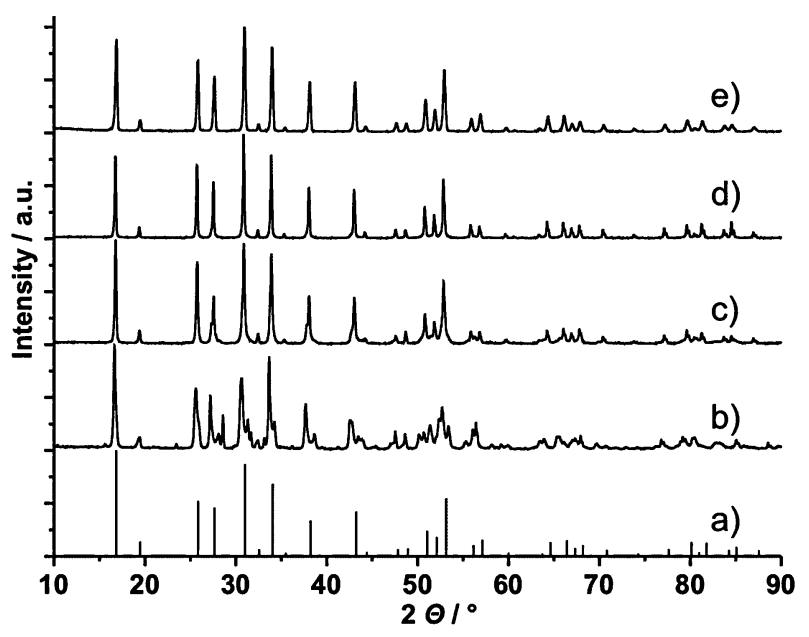

Fig. 2 XRD patterns of LLZO after the final calcination step (a) simulated pattern (JCPDS 80-0457), (b) Al-free synthesis in $\mathrm{Al}$ free crucible, (c) doping with $0.5 \mathrm{wt} \% \gamma-\mathrm{Al}_{2} \mathrm{O}_{3}$ in $\mathrm{Al}$ free crucible, (d) doping with $0.9 \mathrm{wt} \% \gamma-\mathrm{Al}_{2} \mathrm{O}_{3}$ in $\mathrm{Al}$ free crucible, (e) undoped sample prepared in $\mathrm{Al}_{2} \mathrm{O}_{3}$ crucible $(0.9 \mathrm{wt} \% \mathrm{Al}$ content measured by ICP-OES). 

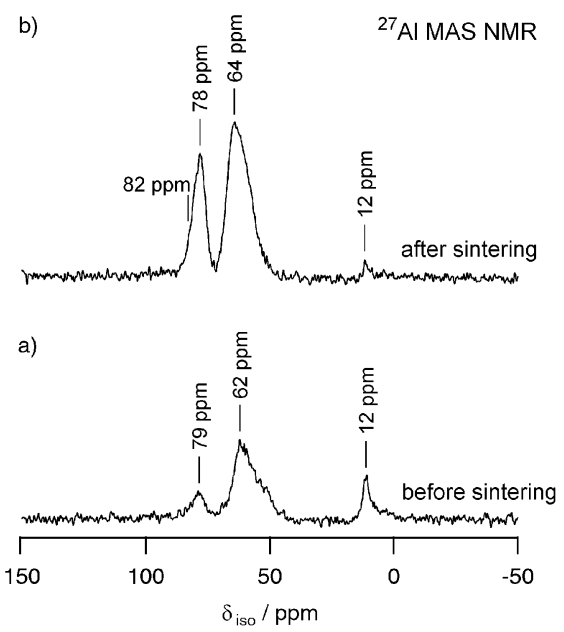

Fig. $3{ }^{27} \mathrm{Al}$ MAS NMR spectra of cubic LLZO before (a) and after sintering (b). The spectra were recorded at a magnetic field of $14.1 \mathrm{~T}$ and referenced to aqueous $\mathrm{Al}\left(\mathrm{NO}_{3}\right)_{3}$. The rotation frequency was $30 \mathrm{kHz}$.

of cubic LLZO before and after sintering at elevated temperatures (see above) are shown. The spectrum obtained after the sintering step is similar to that in ref. 10. Geiger et al. suggest that the signal showing up at a chemical shift of $\delta_{\text {iso }}=12 \mathrm{ppm}$ reflects octahedrally coordinated $\mathrm{Al}$ in $\mathrm{LaAlO}_{3}$ being an

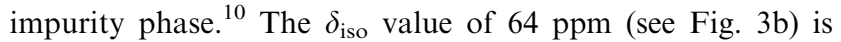
characteristic of $\mathrm{Al}$ ions residing on lattice sites with tetrahedral coordination. Most probably the $\mathrm{Al}$ ions occupy the $\mathrm{Li}$ site $24 d$ in the garnet structure according to the aliovalent doping mechanism $3 \mathrm{Li}^{+} \rightarrow \mathrm{Al}^{3+}$. Multiple-quantum magic angle spinning (MQ-MAS) measurements of Geiger et al. have shown that the asymmetry parameter $\eta$ of the corresponding electric field gradient EFG tensor is close to zero indicating axial symmetry. ${ }^{10}$ Preliminary ${ }^{27} \mathrm{Al}$ MAS measurements on samples with varying $\mathrm{Li}, \mathrm{La}$ and $\mathrm{Al}$ contents indicate that the signal near 80 ppm in fact represents at least two NMR lines. ${ }^{20}$ Obviously, at a sufficiently large $\mathrm{Al}$ content, $\mathrm{Al}^{3+}$ ions occupy various magnetically inequivalent sites in the cubic LLZO structure. NMR lines near $80 \mathrm{ppm}$, which most likely reflect Al ions also occupying sites with tetrahedral coordination, show up for samples of cubic LLZO which are characterized either by a deficit of $\mathrm{La}$ and/or $\mathrm{Zr}^{20}$

It is worth mentioning that the cubic LLZO sample obtained before the sintering step (see the ${ }^{27} \mathrm{Al}$ MAS NMR spectrum in Fig. 3a) is characterized by a Li ion conductivity which is by about two orders of magnitude lower than that reported for cubic LLZO. The low Li conductivity of the non-sintered sample, which is comparable to that of LLZO crystallizing in tetragonal symmetry, has been confirmed by ${ }^{7} \mathrm{Li}$ NMR spin-lattice relaxation measurements revealing a rather low Li diffusivity. ${ }^{21}$ Comparison of the two spectra reveals distinct differences which might help explain the high ion conductivity (see below) of the sintered material from an atomic-scale point of view. Firstly, the sintering step clearly enhances the Al content within LLZO. Secondly, the NMR line intensity at $12 \mathrm{ppm}$ has decreased after the sintering step. Thus, $\mathrm{LaAlO}_{3}$ seems to act as a kind of precursor for the incorporation of $\mathrm{Al}$ into LLZO.

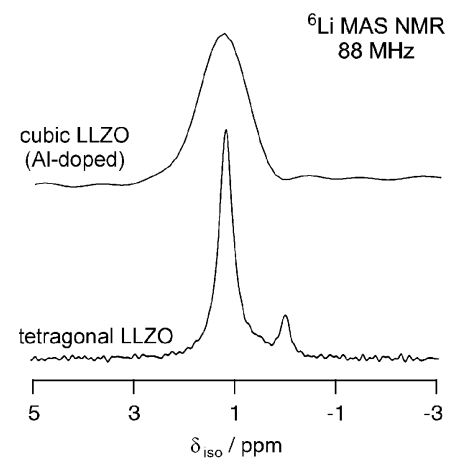

Fig. $4{ }^{6} \mathrm{Li}$ MAS NMR spectra of cubic and tetragonal LLZO. The latter was taken from ref. 21. A $1 \mathrm{M}$ aqueous solution of $\mathrm{LiCl}$ served as reference. The spectrum of cubic LLZO refers to the sample which has been sintered at elevated temperatures; the corresponding ${ }^{27} \mathrm{Al}$ MAS NMR spectrum is shown in Fig. 8b. Note that the tetragonal Al-free sample and its Li dynamics have been investigated in detail by NMR spectroscopy in ref. 21 .

Finally, in Fig. 4 (top) the ${ }^{6} \mathrm{Li}$ MAS spectrum of Al-doped cubic LLZO is shown. Even under MAS conditions with a spinning rate of $30 \mathrm{kHz}$, the ${ }^{6} \mathrm{Li}$ NMR line is comparably broad showing a line width (full width at half maximum, fwhm) of $1.1 \mathrm{ppm}$. This value should be compared with that one of the tetragonal Al-free counterparts (space group $I 4_{1} /$ acd $)^{21}$ (see Fig. 4, bottom).

\section{Crystal structure from neutron and X-ray diffraction}

Our XRD results do not indicate any tetragonal distortion, but show some weak reflections, which can be attributed to a residue of lithium carbonate. These reflexes are more pronounced in the neutron data (Fig. 5) and the content of lithium carbonate can be quantified as $1.8(4) \mathrm{wt} \%$. The comparison of neutron powder diffraction patterns collected at $4 \mathrm{~K}$ and $300 \mathrm{~K}$ indicates the isostructurality of LLZO at both temperatures. All reflexes originate either from cubic LLZO as the main phase or lithium carbonate as the residual phase. Different structure models were considered and simulated ${ }^{7,10,22}$ which, however, did not reveal a satisfactory description of the Bragg intensities mainly due to underestimated isotropic displacement parameters and differences in lithium occupations. After the adjustment of the models, the best results have been obtained with the model proposed by Geiger et al. ${ }^{10}$ Aluminium was not taken into account in their structure model, as its position in the lattice cannot easily be determined by X-ray diffraction. In fact, in the presence of relatively heavy lanthanum and zirconium atoms the location of "light" lithium and aluminium atoms is cumbersome. Moreover, the neutron scattering length $b$ for aluminium is rather low and therefore small concentrations can be hardly quantified using neutron powder diffraction. However, the ${ }^{27} \mathrm{Al}$ MAS NMR spectra show that about $70 \%$ of the aluminium is located on the lithium sites with $24 d$ symmetry which was included into our structure simulation. Placing $\mathrm{Al}$ on the $24 d$ position did not improve the residual intensities in the difference between observed and calculated diffraction patterns, but affects the lithium occupation on the $24 d$ site. Without aluminium $g_{\mathrm{Li}(24 d)}$ has been found to be $42(2) \%$, whilst $g_{\operatorname{Li}(24 d)}=52(2) \%$ was 

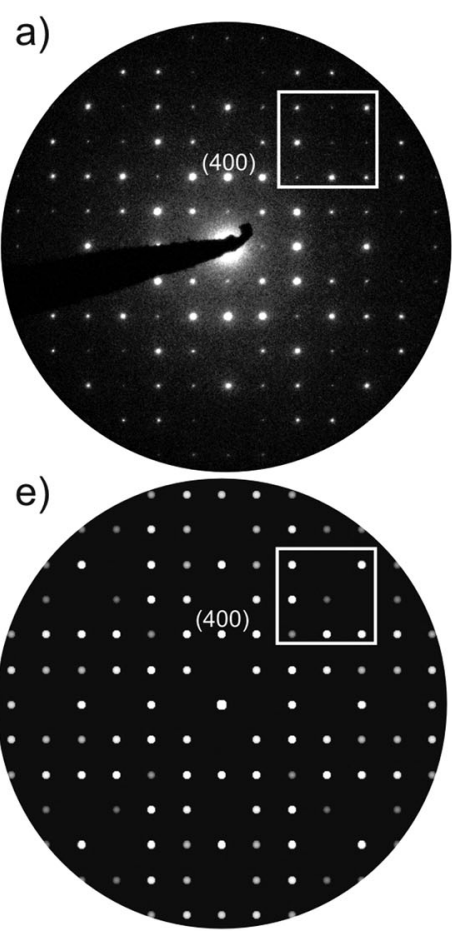

c)

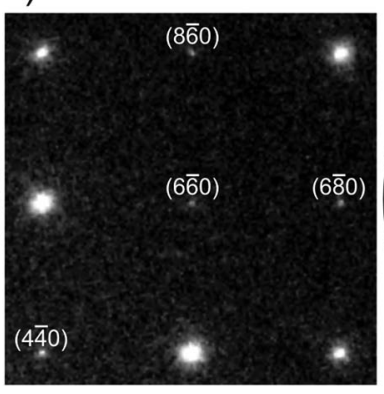

f)

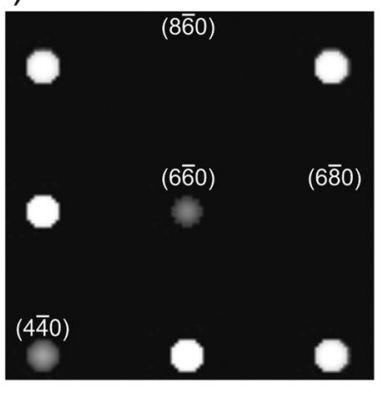

g)
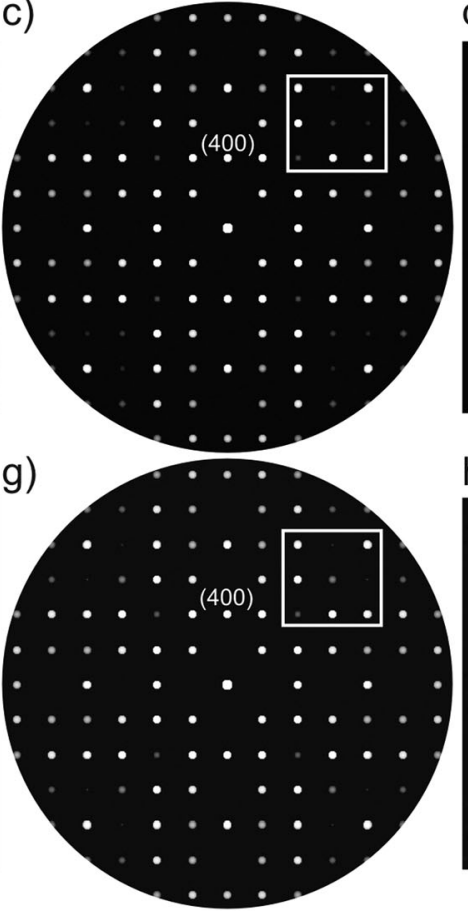

d)

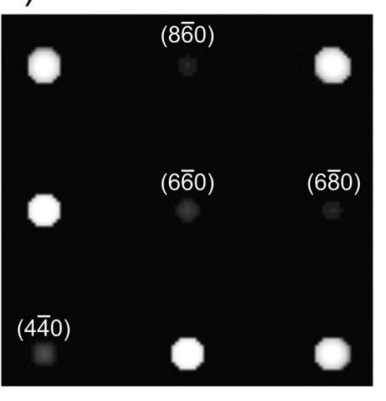

h)

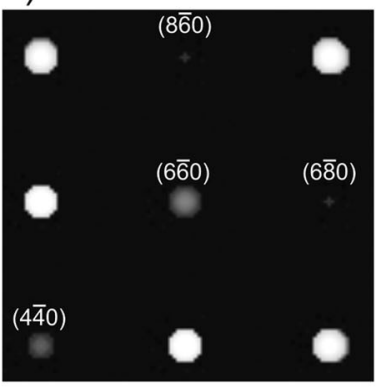

Fig. 5 (a) Experimental and (c) simulated PED patterns of LLZO along [001] assuming the cubic structural model from Table 1. (e) Simulated PED pattern of cubic LLZO according to ref. 9. (g) Simulated PED images of cubic LLZO using the structural model from ref. 10. (b), (d), (f) and (h) Enlarged areas of the marked regions in (a), (c), (e) and (g). The differences between the simulated and experimental PED patterns are clearly seen, with the experimental pattern most convincingly agreeing with the simulated image in (c) (our structure model from Table 1).

determined assuming the presence of $\mathrm{Al}$ with $g_{\mathrm{Al}(24 d)}=6.53 \%$. In the latter case the overall lithium content $x$ in $\mathrm{Li}_{x} \mathrm{La}_{3} \mathrm{Zr}_{2} \mathrm{O}_{12}$ has been determined to be $6.05 \pm 0.25$, which agrees with the proposed heterovalent substitution mechanism $3 \mathrm{Li}^{+} \leftrightarrow \mathrm{Al}^{3+}$ (see above). ${ }^{10}$ The best fits to the neutron diffraction data collected at $4 \mathrm{~K}$ and $300 \mathrm{~K}$ were obtained with the parameters listed in Table 1 and the results of the Rietveld refinements are shown in Fig. 5. Anomalously large isotropic displacement parameters have been noticed for both lithium sites in LLZO already at low temperatures, which might be attributed to static lithium disorder. An application of the split site model to $\mathrm{Li}$ on the $24 d$ atomic site did neither lead to a stable fit nor a significant improvement of the fit residuals. The comparison of results obtained at $4 \mathrm{~K}$ and $300 \mathrm{~K}$ indicates a minor influence of the temperature on the crystal structure of LLZO which is quite typical for garnet-like structures. ${ }^{23,24}$ Heating from $4 \mathrm{~K}$ to $300 \mathrm{~K}$ results in the elongation of the lattice parameter $a$ by $0.223 \%$ with very small atomic movements involved. This means that only a small shift of Li2 in the $a_{1}$ direction of the cubic lattice takes place, whereas the coordinates for the other atoms in LLZO remain almost unchanged within the limits of their estimated standard deviations. Temperature increase causes a pronounced rise of displacement parameters on all atomic sites, but the major increase (ca. 50\%) was observed for $\mathrm{Li}$ on the $96 \mathrm{~h}$ atomic site and ( $c a .30 \%$ ) for $\mathrm{Li}$ on $24 d$. A strong increase of the displacement parameter indicates a development of dynamic lithium disorder on the background of the static one. The analysis of the temperature evolution of the anisotropic displacement parameters for lanthanum, zirconium and oxygen indicates their evolution from elliptic towards spherical shape with nearly unchanged spatial orientation. This might correspond to a temperature stability of the framework built by these elements. A closer look on the disorder details by examination of differential Fourier maps indicates the presence of quite broad minima around Li (24d) with a stripe-like shape penetrating the garnet lattice. This anomaly can be related to the observed very high displacement parameters. However, details of such stripes with negative densities were sufficiently smeared out due to termination effects. Therefore, further examinations of the disorder have been performed using the maximum entropy method (MEM) ${ }^{26}$ which can estimate non-zero structure factors for high-Q reflections (typically excluded in the analysis of the powder diffraction data). This feature makes the termination effect in MEM analysis less pronounced in comparison to Fourier synthesis, thus yielding less noisy three-dimensional distributions of electron/nuclear densities. Therefore, the MEM technique has been successfully used to a diversity of scientific problems either concerning disorder in $\mathrm{Sr}_{9.3} \mathrm{Ni}_{1.2}\left(\mathrm{PO}_{4}\right)_{7}{ }^{27}$ and in $\mathrm{Rb}_{4} \mathrm{Cu}_{16} \mathrm{I}_{7.2} \mathrm{Cl}_{12.8}{ }^{28}$ or experimental visualization of lithium diffusion in $\mathrm{Li}_{x} \mathrm{FePO}_{4}{ }^{29}$ and $\mathrm{La}_{9.69}\left(\mathrm{Si}_{5.70} \mathrm{Mg}_{0.30}\right) \mathrm{O}_{26.24}{ }^{30}$

The negative nuclear densities obtained after the MEM analysis of datasets collected at $4 \mathrm{~K}$ and $300 \mathrm{~K}$ are shown in Fig. 6. They correspond to stripes observed in differential Fourier maps and might be related to the lithium diffusion pathway (as lithium is the only negative neutron scatterer in LLZO), where all lithium atoms take part in the diffusion process. Rise of the temperature from $4 \mathrm{~K}$ to $300 \mathrm{~K}$ unambiguously indicates an enhancement of Li-ion diffusivity, which corresponds to the increase of the ionic conductivity. 
Table 1 Refined structural parameters of cubic LLZO at $T=4 \mathrm{~K}$ and $T=300 \mathrm{~K}$ as deduced from Rietveld refinement with FullProf. The space group is $I a \overline{3} d$ (No. 230). Displacement parameters for lithium were modelled in isotropic approximation. Numbers in parentheses give the statistical deviations of the last significant digit

$T=4$ K, Lattice parameter: $a=12.9438(2) \AA$

\begin{tabular}{|c|c|c|c|c|c|c|}
\hline Atom site & $x / a_{1}$ & $y / a_{2}$ & $z / a_{3}$ & $u_{\mathrm{iso}}=u_{\mathrm{eq}} / \AA^{2}$ & $u_{11}, u_{22}, u_{33}, u_{12}, u_{13}, u_{23} / \AA^{2}$ & Occ. $g$ \\
\hline $\mathrm{Li} 1,24 d$ & $1 / 8$ & 0 & $1 / 4$ & $0.041(6)$ & - & $0.54(2)$ \\
\hline $\mathrm{All}, 24 d$ & $1 / 8$ & 0 & $1 / 4$ & $0.041(6)$ & - & $0.0653^{c}$ \\
\hline $\mathrm{Li} 2,96 h$ & $0.0980(7)$ & $0.6859(7)$ & $0.5764(7)$ & $0.020(4)$ & - & $0.37(1)$ \\
\hline $\mathrm{La}, 24 c$ & 0 & $1 / 4$ & $1 / 8$ & $0.0074(6)$ & $\begin{array}{l}0.0065(5), 0.0065(5), 0.0091(10) \\
0.0039(6), 0.00000,0.00000\end{array}$ & 1.0 \\
\hline $\mathrm{Zr}, 16 a$ & 0 & 0 & 0 & $0.0067(3)$ & $\begin{array}{l}0.0067(3), 0.0067(3), 0.0067(3) \\
0.0006(5), 0.0006(5), 0.0006(5)\end{array}$ & 1.0 \\
\hline O, $96 h$ & $-0.03173(8)$ & $0.05463(9)$ & $0.14951(8)$ & $0.0105(6)$ & $\begin{array}{l}0.0120(6), 0.0137(7), 0.0058(5) \\
0.0017(5),-0.0013(4),-0.0037(5)\end{array}$ & 1.0 \\
\hline
\end{tabular}

$T=300 \mathrm{~K}$, Lattice parameter: $a=12.9727(2) \AA$

\begin{tabular}{|c|c|c|c|c|c|c|}
\hline Atom site & $x / a_{1}$ & $y / a_{2}$ & $z / a_{3}$ & $u_{\text {iso }}=u_{\text {eq }} / \AA^{2}$ & $u_{11}, u_{22}, u_{33}, u_{12}, u_{13}, u_{23} / \AA^{2}$ & Occ. $g$ \\
\hline $\mathrm{Lil}, 24 d$ & $1 / 8$ & 0 & $1 / 4$ & $0.054(5)$ & - & $0.54^{b}$ \\
\hline $\mathrm{All}, 24 d$ & $1 / 8$ & 0 & $1 / 4$ & $0.054(5)$ & - & 0.0653 \\
\hline $\mathrm{Li} 2,96 h$ & $0.1004(8)$ & $0.6853(8)$ & $0.5769(8)$ & $0.031(3)$ & - & $0.37^{b}$ \\
\hline $\mathrm{La}, 24 c$ & 0 & $1 / 4$ & $1 / 8$ & $0.0106(6)$ & $\begin{array}{l}0.0095(4), 0.0095(4), 0.0128(9) \\
0.0035(6), 0.00000,0.00000\end{array}$ & 1.0 \\
\hline $\mathrm{Zr}, 16 a$ & 0 & 0 & 0 & $0.0095(3)$ & $\begin{array}{l}0.0095(3), 0.0095(3), 0.0095(3) \\
0.0005(5), 0.0005(5), 0.0005(5)\end{array}$ & 1.0 \\
\hline $\mathrm{O}, 96 h$ & $-0.03161(8)$ & $0.05454(9)$ & $0.14940(8)$ & $0.0148(6)$ & $\begin{array}{l}0.0170(6), 0.0179(6), 0.0094(5) \\
0.0018(5),-0.0006(4),-0.0026(5)\end{array}$ & 1.0 \\
\hline
\end{tabular}

Fit residuals: $R_{\mathrm{p}}=2.22 \%, R_{\mathrm{wp}}=2.79 \%, R_{\exp }=0.94 \%$

${ }^{a}$ Taken from NMR experiments. ${ }^{b}$ Fixed to the value obtained at $4 \mathrm{~K}$.
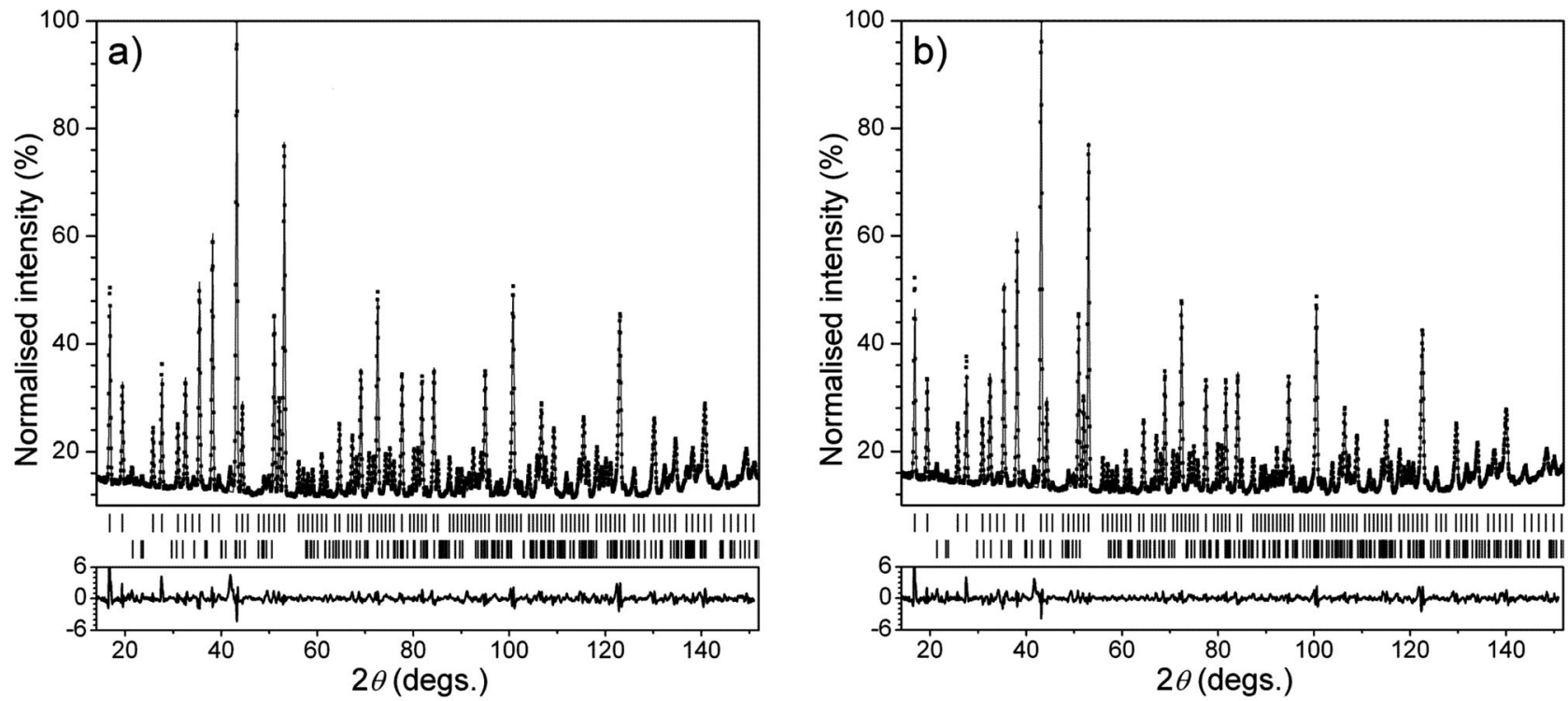

Fig. 6 Results of Rietveld refinements for neutron powder diffraction data at $4 \mathrm{~K}$ (a) and $300 \mathrm{~K}$ (b) for LLZO ( $\lambda=1.5482 \AA$ ). Calculated positions of Bragg reflections are shown by vertical tick marks, where the top row of the tick marks corresponds to LLZO, whilst the lower ones describe the peak positions for lithium carbonate. Differences between observed and calculated pattern are shown below.

It is noteworthy that the structural investigations were carried out on the LLZO powder before sintering. Although the ${ }^{27} \mathrm{Al}$ NMR results show changes of the Al distribution on different crystallographic sites during sintering which seems to have a significant effect on the Li diffusivity we do not believe that this significantly changes the results of the diffraction investigations. As already mentioned it is quite cumbersome to refine $\mathrm{Al}$ and $\mathrm{Li}$ positions with a high level of reliability. Therefore, from a crystallographic point of view, the small structural changes detected by NMR after sintering might only lead to an increase of the $\mathrm{Li}$ displacement parameters. The small changes in the occupations of $\mathrm{La}$ and $\mathrm{Zr}$ positions by $\mathrm{Al}$ will hardly be detectable by diffraction methods. As the disorder of $\mathrm{Li}$ atoms is already quite high, an additional 
increase will be within the limit of reasonably refineable displacement parameters. Nevertheless, this will be a subject for further investigations.

\section{PED and HRTEM results}

In the last decade, PED has become a powerful technique for the advanced characterization of materials. The superior advantage of the PED method over the SAED technique is its ability to reduce dynamical effects arising due to multiple scattering in the transmission electron microscope. Dynamical effects in SAED patterns make the distinction between two phases difficult, particularly, if the phases are structurally and chemically related, as it is the case for cubic and tetragonal LLZO (see ESI $\dagger$, Fig. SX1). In this case, the diffraction patterns differ only by the absolute values of diffracted intensity. Recently, PED was successfully applied for the distinction of polymorphs occurring in bulk materials and thin films, e.g. of quartz ${ }^{31}$ and $\mathrm{Ag}_{2} \mathrm{Se}^{32}$ respectively. In the present work, we compare our results from PED examinations on single microcrystals with those from neutron powder diffraction. Fig. 7a displays the experimental PED pattern of a LLZO sample containing $0.9 \mathrm{wt} \%$ of $\mathrm{Al}$ and the simulated PED patterns based on the cubic and tetragonal LLZO along zone axis [001] (Fig. 7b and c, respectively). The differences in the simulated intensities of the reflections $h 00$ with $h=2 n$ are obvious.

For the cubic phase, the $h 00$ reflections with $h \neq 4 n$ are kinematically forbidden (Fig. 7b). However, since the PED only reduces dynamical effects, the serial reflection conditions based on the $4_{1}$ screw axis can be violated by residual dynamical effects. Thus, the significant intensity of the (200) reflection in the experimental PED pattern (Fig. 7a) can be rationalized, even for the cubic structure. The dynamical effects decrease with increasing $h$, thus, the intensity of the reflections (600) and (10 00$)$ are zero in the experimental and simulated patterns (Fig. 7a and b). In the case of the tetragonal LLZO, the integral reflection condition $h 00$ with $h=2 n$ is consistent with the occurrence of strong reflections (600) and $\left(\begin{array}{lll}10 & 0 & 0\end{array}\right), c f$. the simulated pattern in Fig. 7c. Simulated patterns based on the tetragonal and cubic polymorphs also indicate differences in the intensities of (440) and (660) reflections, $c f$. line profiles in Fig. 7. Thus, the experimental PED pattern is better described by the cubic structure of LLZO. It should be noted that we have tested different structural models for PED simulations obtained from neutron powder diffraction. We found that calculated PED patterns using the structural model in Table 1 show the best match with our experimental PED data. In addition, the calculated PED patterns using the structural model for LLZO of Table 1 match even better as the PED images calculated based on the models for the cubic LLZO ${ }^{8,10}$ (see e.g. Fig. 8).

Even when adjusting low dose settings, the LLZO sample was extremely unstable under the imaging conditions used for HRTEM. Thus, a complete series of images with variable focus values could not be recorded without initiating considerable loss of structural ordering. Fig. 9a and b show experimental and Fourier-filtered HRTEM micrographs for the first overfocused image of a focus series, zone axis [111]. The simulated micrograph of Fig. 9c convincingly agrees confirming again our assignment to the cubic structure given in Table 1. However, after a few seconds the structure is completely amorphized, just leaving crystalline islands embedded within an amorphous matrix, $c f$. complete focus series in $\mathrm{ESI} \dagger$, Fig. SX2.

EDX investigations showed a homogeneous distribution of Al in the LLZO sample within the experimental errors. Particularly, no Al-rich regions were found in the LLZO specimen (see ESI $\dagger$, Fig. SX3).

\section{Electrochemical results}

Electrochemical measurements were performed to determine the ionic and electronic conductivity of the cubic LLZO. The total conductivity was measured both by ac impedance spectroscopy and four electrode dc measurements. The electronic conductivity was determined by the Hebb-Wagner technique. ${ }^{18,19}$ Impedance measurements with $\mathrm{Au}$ electrodes on LLZO known from the literature show two semicircles and a low frequency tail. These were interpreted to be corresponding to the bulk, grain boundary and electrode contributions, respectively. ${ }^{6}$ Our impedance measurements performed with
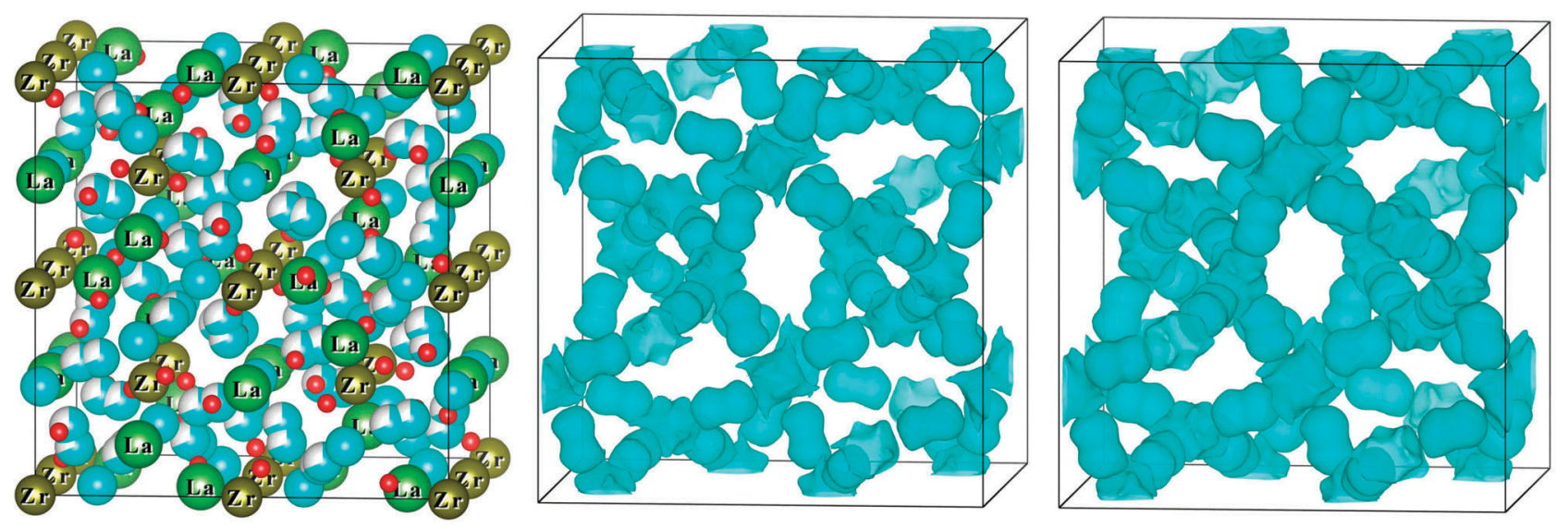

Fig. 7 Structural model of cubic LLZO (left, atoms shown by cyan and red correspond to lithium and oxygen, respectively) and negative scattering neutron density maps determined at $4 \mathrm{~K}$ (middle) and $300 \mathrm{~K}$ (right) by MEM. Negative scattering neutron density maps are shown with a equidensity level of $0.035 \mathrm{fm} \AA^{-3}$. Data visualization has been performed using the program $\mathrm{VESTA}^{25}$ 


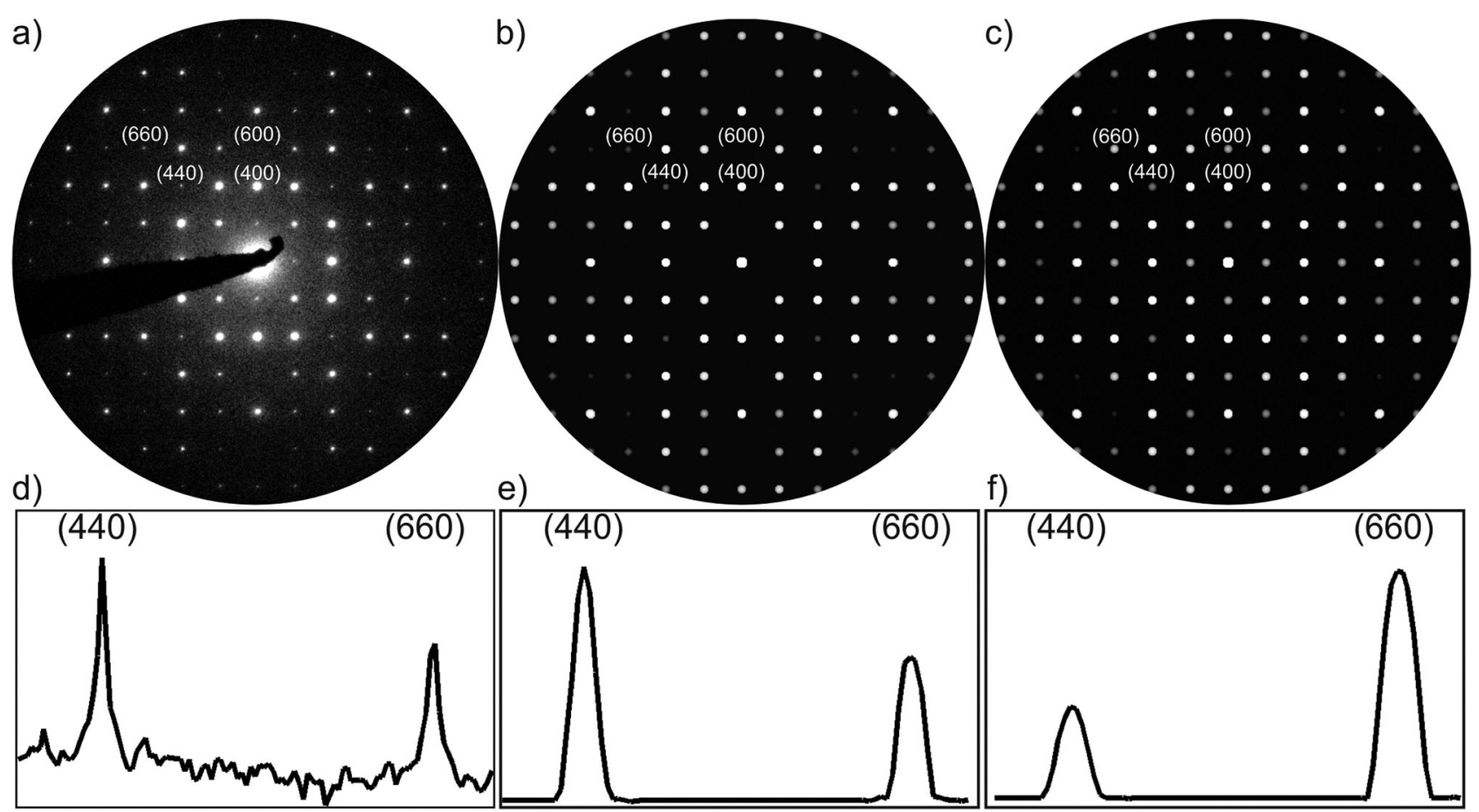

Fig. 8 (a) Experimental and (b) simulated PED patterns of LLZO along [001]. For the simulation of (b) we used the cubic structure model presented in Table 1. (c) Simulated PED images of tetragonal LLZO based on the structure model from ref. 7. (d)-(f) Line profiles taken from (440) and (660) reflections in (a)-(c). The differences in the intensities of (600), (440) and (660) reflections are clearly seen. The experimental pattern fits better to the cubic LLZO.

a)

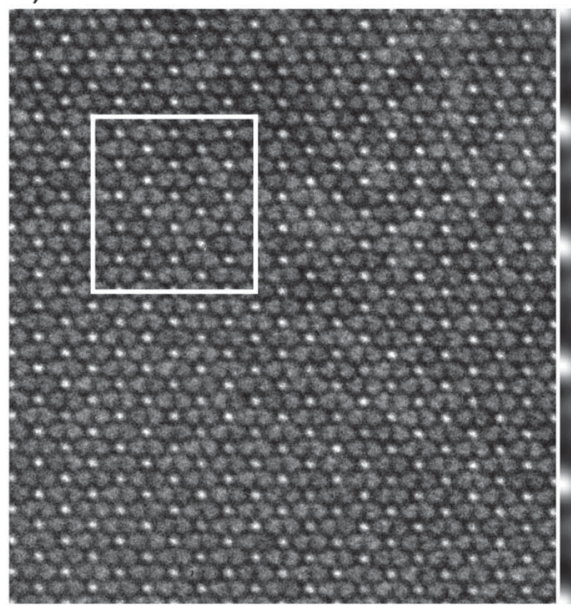

$5 \mathrm{~nm}$ b)

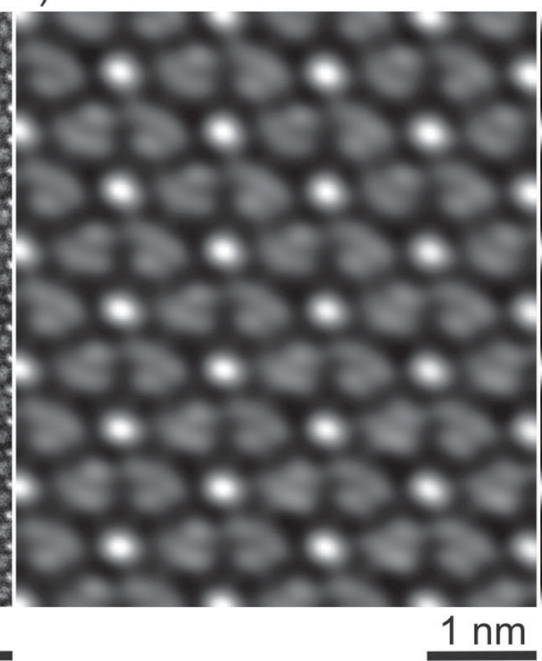

c)

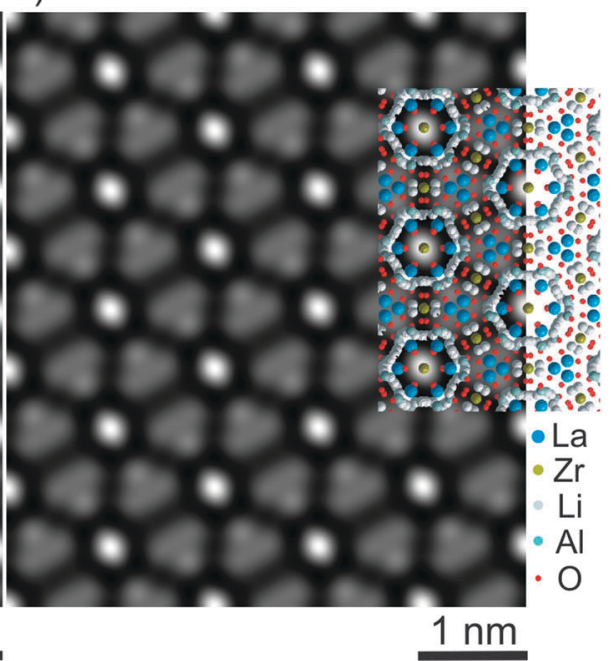

Fig. 9 (a) Experimental HRTEM micrograph of LLZO along [111] viewing direction. (b) Fourier-filtered HRTEM image of the region marked in (a). (c) Simulated HRTEM image (defocus $90 \mathrm{~nm}, t=9 \mathrm{~nm}$, 2-fold astigmatism estimated to $10 \mathrm{~nm}$ ) based on the crystal structure model of LLZO from Table 1 (inserted).

Li electrodes show only one semicircle for the total resistivity of the LLZO pellet and a second semicircle instead of the tail. The appearance of an additional semicircle when using $\mathrm{Li}$ electrodes can be expected since $\mathrm{Li}$ electrodes can reversibly incorporate Li ions from the LLZO, so the charge transfer resistance and the electrode capacity should give rise to an additional semicircle in the impedance measurements explaining the appearance of a second semicircle instead of the low frequency tail. Still it was surprising that our samples only showed one semicircle for the LLZO contribution so we took a detailed look into the impedance data to be sure that there was no misinterpretation. Fig. 10a shows an example of a typical Nyquist plot measured on a LLZO pellet between Li electrodes. Impedance data were fitted with an equivalent circuit $(\mathrm{RC})(\mathrm{RQ})$ or $(\mathrm{RQ})(\mathrm{RQ})$ depending on whether the respective sample showed a depressed high frequency semicircle or not. 
From the fit to this measurement the first high frequency semicircle resistance and capacity were determined to be 20.4 $\mathrm{k} \Omega$ and $0.38 \mathrm{nF}$, respectively. The second low frequency semicircle resistance and capacity result in $13.3 \mathrm{k} \Omega$ and $0.15 \mu \mathrm{F}$, respectively. These relatively high capacity values are typical for grain boundaries or electrodes of polycrystalline ceramics. ${ }^{33}$ As both semicircles yield to comparable resistances as supposed for the bulk and grain boundary contributions in cubic LLZO, ${ }^{6}$ some additional experiments were carried out to clarify the origin of the impedance contributions when using Li electrodes. Fig. 10b shows a Nyquist plot of a sample measured once with $\mathrm{Au}$ and once with reversible $\mathrm{Li}$ electrodes. The $\mathrm{Au}$ electrodes were gas phase deposited by thermal evaporation, whereas the Li electrodes were pressed
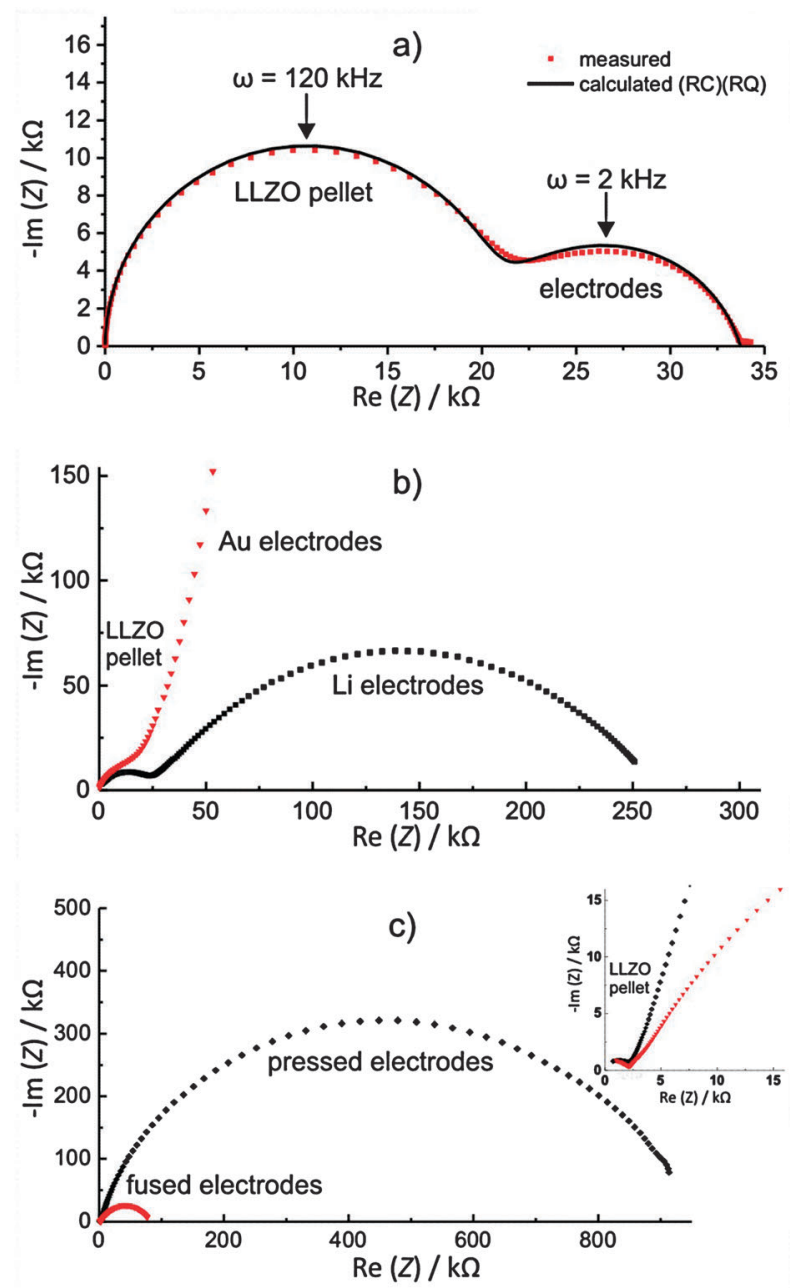

Fig. 10 Room temperature impedance spectra of different LLZO samples with various electrodes (a) impedance spectrum of a LLZO pellet doped with $0.9 \mathrm{wt} \% \mathrm{Al}$. The pellet size was $5.3 \mathrm{~mm}$ diameter and $16.2 \mathrm{~mm}$ length. The pellet was sandwiched between two Li electrodes (frequency range from $7 \mathrm{MHz}$ to $50 \mathrm{mHz}$ at $20 \mathrm{mV}$ amplitude). An equivalent circuit (RC)(RQ) was used to fit the impedance data. (b) Impedance spectra of a LLZO pellet doped with $0.9 \mathrm{wt} \% \mathrm{Al}$ measured with blocking $\mathrm{Au}$ and reversible $\mathrm{Li}$ electrodes in a frequency range $7 \mathrm{MHz}$ to $1 \mathrm{~Hz}$ at $20 \mathrm{mV}$. (c) Impedance spectra of a LLZO pellet doped with $0.9 \mathrm{wt} \% \mathrm{Al}$ measured with $\mathrm{Li}$ electrodes pressed and fused on the pellet (frequency range $7 \mathrm{MHz}$ to $1 \mathrm{~Hz}$ at $20 \mathrm{mV}$ ). mechanically on the pellet. The measurement with the $\mathrm{Au}$ electrodes shows a semicircle merging into a low frequency tail. The low frequency tail is caused by an almost solely capacitive behaviour of the $\mathrm{Au}$ electrodes indicating their blocking nature under the given experimental conditions. The measurement with $\mathrm{Li}$ electrodes leads to two semicircles proving that the second semicircle corresponds to the $\mathrm{Li}$ electrodes charge transfer resistance and capacity. This also clarifies that our samples only show one semicircle representing the total resistivity of the LLZO pellet. Murugan et al. reported that bulk and grain boundary contributions could only be resolved at low temperatures, ${ }^{6}$ i.e., in their case room temperature and slightly above. In the present case even at $-40{ }^{\circ} \mathrm{C}$ only one semicircle representing the total resistivity of the sample is observed. As shown in Fig. 10c the charge transfer resistance of the $\mathrm{Li}$ electrodes depends strongly on the preparation method of the $\mathrm{Li}$ electrodes. Two impedance measurements of the same sample, in one case with $\mathrm{Li}$ electrodes gently pressed on the pellet and in the second case with $\mathrm{Li}$ electrodes fused on the pellet, were performed. The different preparation methods change the charge transfer resistance almost by one order of magnitude while the pellet resistance remains unchanged as seen in the inset. We did not find any evidence for a reaction of the LLZO with Li metal which might have shown up as an additional impedance during our measurements. It has to be noted that the charge transfer resistance is not negligible and may play a role when LLZO is applied as a solid electrolyte in battery cells with $\mathrm{Li}$ metal electrodes. The total voltage drop across a cell is the sum of the charge transfer resistances at the electrode interfaces and the voltage drop along the electrolyte bulk itself. Therefore further investigations on the charge transfer resistances between different electrode materials and preparation procedures are in progress.

In addition to ac impedance we used four electrode dc measurements to determine the conductivity of the samples under continuous current load. By using the four electrode dc technique the bulk resistance of the sample (as a sum of grain and grain boundary contributions) can be determined without electrode overvoltages. The results of the dc conductivities are shown together with ac data in Fig. 11. Comparing the conductivities of samples with different aluminium contents it is clearly visible that the conductivity depends on the aluminium content in the samples. The highest conductivity of $3 \times 10^{-4} \mathrm{Scm}^{-1}$ to $4 \times 10^{-4} \mathrm{Scm}^{-1}$ at $25^{\circ} \mathrm{C}$ were found for samples containing $0.9 \mathrm{wt} \% \mathrm{Al}$ prepared either by synthesis in $\mathrm{Al}_{2} \mathrm{O}_{3}$ crucibles or by intentional $\gamma-\mathrm{Al}_{2} \mathrm{O}_{3}$ doping. The Arrhenius activation energy is determined to be $0.34 \mathrm{eV}$. The conductivity and activation energy data agree well with results from Murugan et $a l .{ }^{6}$ who did not report on any aluminium in their samples and did not give information on the impurity level at all. The $0.5 \mathrm{wt} \% \mathrm{Al}$ containing sample $\left(\gamma-\mathrm{Al}_{2} \mathrm{O}_{3}\right.$ doped) shows a slightly lower conductivity of $1 \times 10^{-4} \mathrm{Scm}^{-1}$ but the same activation energy of $E_{\mathrm{a}}=0.34 \mathrm{eV}$. The Al-free sample with tetragonal crystal structure is characterized by a two orders of magnitude lower conductivity of $2 \times$ $10^{-6} \mathrm{Scm}^{-1}$ at $25{ }^{\circ} \mathrm{C}$ and an activation energy $E_{\mathrm{a}}=$ $0.49 \mathrm{eV}$. The latter value is in good agreement with that reported for a tetragonal sample which has recently been 


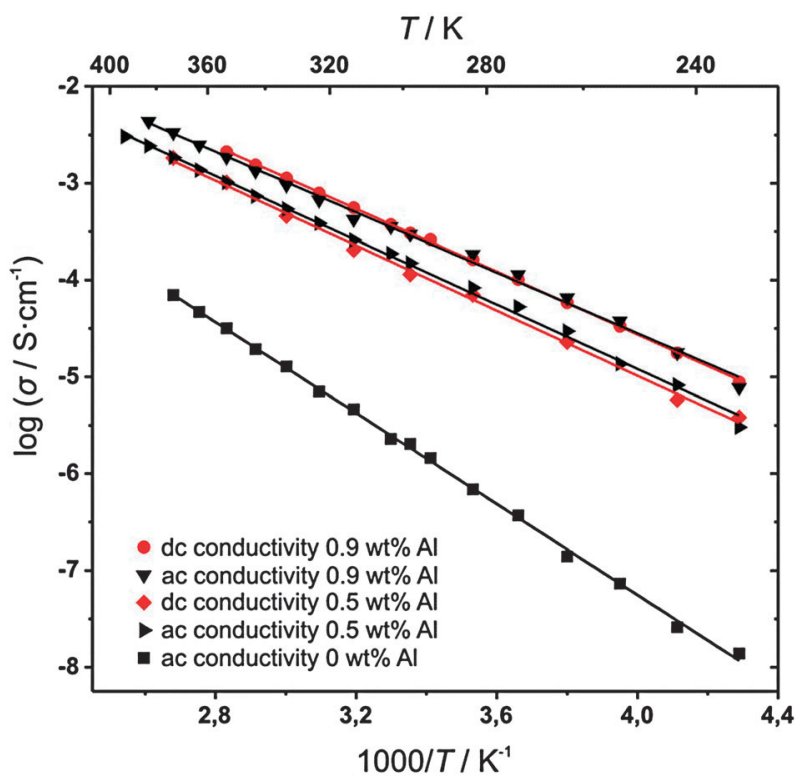

Fig. 11 Temperature dependence of the conductivity of LLZO samples with different $\mathrm{Al}$ contents. Al-containing samples have been studied by two electrode ac (impedance) as well as four electrode de techniques. Note, for the sake of readability $\log \sigma$ vs. 1000/T is shown. The activation energies given in the text were calculated from the slope of $\ln (\sigma T)$ vs. $1000 / T$ remembering that $\ln (\sigma T)=$ $\ln \sigma_{0}-E_{\mathrm{a}} / R T$.

investigated by NMR spectroscopy. ${ }^{21}$ The electronic conductivity of cubic LLZO, which controls the self-discharge of a lithium battery and therefore is of great interest, was measured with a Hebb-Wagner type cell arrangement with one reversible and one blocking electrode (Fig. 1c). In optimal cases Hebb-Wagner measurements can be used to determine the individual electron hole and electron partial conductivities as a function of the Fermi level (i.e. as a function of the lithium metal content in the present case).

When the cell is polarized, as can be seen in Fig. 1c, a high mixed ionic and electronic transient current is rapidly decreasing and finally reaching a steady state electronic current. The steady state current under ion-blocking conditions can only be due to electron and hole conduction in the material as no Li ions can be delivered from the blocking gold electrode. Prior to the polarization experiments the open circuit voltage of the cell shown in Fig. 1c was approximately $2 \mathrm{~V}$ which is probably due to formation of a lithium gold alloy at the LLZO Au interface ${ }^{34}$ prior to the measurement. Lithium activity gradients in the sample may also be a reason as well as capacitive effects of the high resistance cell. (Note that the low electronic conductivity leads to an electronic resistance in the order of $10^{10} \Omega$ ). Fig. 12 shows the potential dependent mean conductivities measured in the range of typical battery operating voltage. As described in detail in the literature on the Wagner-Hebb technique, the increasing voltage leads to a corresponding decrease of the chemical potential of $\mathrm{Li}$ at the $\mathrm{Au}$ electrode. This changes the electron and hole concentrations in LLZO. Therefore, the overall electronic partial conductivity increases with increasing polarization voltages. Over the potential range of $2.5 \mathrm{~V}-4.5 \mathrm{~V}$ the electronic conductivity takes values in the range from

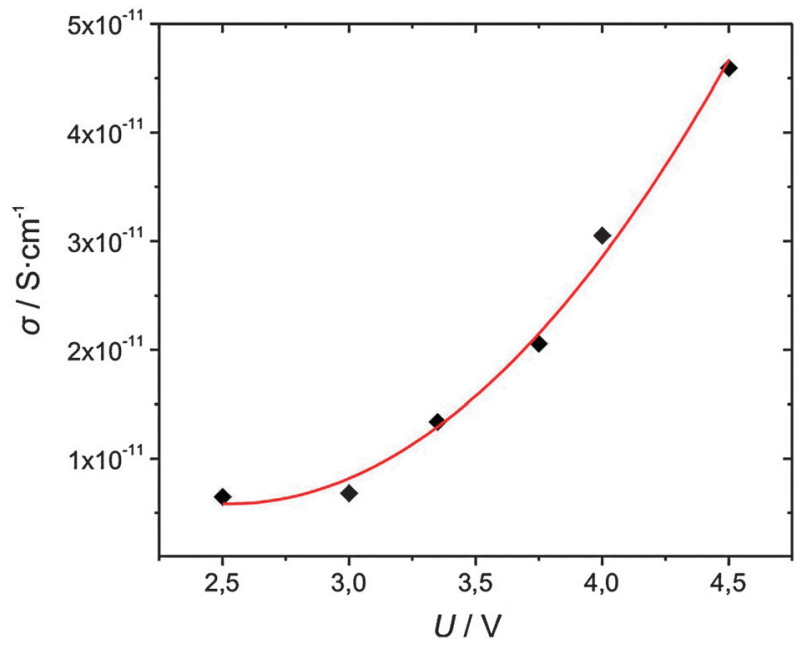

Fig. 12 Partial electronic conductivity of a cubic LLZO pellet $v s$. Hebb-Wagner cell voltage at room temperature. The solid line is only a guide to the eye.

$5 \times 10^{-12} \mathrm{Scm}^{-1}$ to $5 \times 10^{-11} \mathrm{Scm}^{-1}$. With the total conductivity of $3 \times 10^{-4} \mathrm{Scm}^{-1}$ the electronic and ionic transference numbers

$$
t_{\mathrm{e}^{-}}=\frac{\sigma_{\mathrm{e}^{-}}}{\sigma_{\mathrm{total}}} \text { and } t_{\mathrm{Li}^{+}}=\frac{\sigma_{\mathrm{Li}^{+}}}{\sigma_{\mathrm{total}}}
$$

result in values between $2 \times 10^{-8}-2 \times 10^{-7}$ and 0.99999998-0.9999998, respectively. This clearly shows that cubic Al-doped LLZO is an almost exclusive $\mathrm{Li}$ ion conducting material having great potential as an electrolyte for lithium-ion batteries. We note that the increase of the electronic conductivity with increasing potential is due to growing hole conduction.

\section{Solid-state NMR measurements}

In addition to impedance spectroscopy we used various NMR techniques to probe Li dynamics in a sintered sample of garnet-like LLZO crystallizing with cubic symmetry. In Fig. 13 the central transitions (see below) of a typical set of ${ }^{7} \mathrm{Li}$ NMR spectra of cubic LLZO doped with $0.9 \mathrm{wt} \%$. Al are shown. The corresponding ${ }^{27} \mathrm{Al}$ MAS NMR spectrum is shown in Fig. 3b. The ${ }^{7} \mathrm{Li}$ NMR spectra were recorded at various temperatures at a resonance frequency of $155.5 \mathrm{MHz}$. The broad NMR line, which is observed at the very low temperatures, can be well represented by a Gaussian reflecting the distribution of resonance frequencies due to non-averaged dipole-dipole interactions of the $\mathrm{Li}$ nuclei. Here, the associated line width $\delta$ (full width at half maximum) of this so-called rigid lattice regime is given by $\delta_{\mathrm{rl}}=9.7(2) \mathrm{kHz}$. In this temperature range the mean $\mathrm{Li}$ jump rate $1 / \tau$ is much smaller than the spectral width $\delta_{\mathrm{rl}}$, i.e., $1 / \tau \ll 10^{3} \mathrm{~s}^{-1}$. With rising temperature $1 / \tau$ increases resulting in an averaging of homonuclear dipolar interactions. Consequently, the NMR line increasingly narrows, finally reaching the width $\delta_{\text {en }}$ which is solely governed by inhomogeneities of the external magnetic field used (regime of extreme narrowing). Interestingly, in the case of cubic LLZO doped with $\mathrm{Al}$ a heterogeneous motional narrowing $(\mathrm{MN})$ is observed. At intermediate temperatures 
the NMR line partially narrows resulting in a two-component line shape, i.e., the broad rigid-lattice line (see above) is superimposed by a motionally narrowed Lorentzian-shaped component. ${ }^{35}$ Recently, the same behaviour has been observed by Koch et al. who studied the Li dynamics in the garnet $\mathrm{Li}_{5} \mathrm{La}_{3} \mathrm{Nb}_{2} \mathrm{O}_{12} \cdot{ }^{36}$ It is worth mentioning that the spectra shown in Fig. 13 were simply obtained by Fourier transformation of a free induction decay recorded after excitation with a $90^{\circ}$ pulse. Thus, quadrupole intensities arising from the interaction of the spin- $3 / 2$ probe nucleus with electric field gradients produced by the electric charge distribution in the neighbourhood of the Li nuclei are largely suppressed. These can be made visible by using appropriate echo pulse sequences. ${ }^{35,37}$ Usually, when low temperatures are regarded, such interactions are represented by a broad Gaussian line of low intensity characterized by a width of some tens of $\mathrm{kHz} \cdot{ }^{21,36}$ As an example, a stimulated echo NMR spectrum is shown in Fig. 13e which has been recorded using the Jeener-Broekaert echo sequence. ${ }^{38}$ In the present case, we will exclusively focus on the ${ }^{7} \mathrm{Li} \mathrm{NMR}$ central transition of LLZO. In contrast to other garnet-like oxides, in the case of cubic LLZO two-phase NMR spectra show up at temperatures as low as $200 \mathrm{~K}$ indicating a spin ensemble of rapidly diffusing Li ions (see Fig. 13). As an example, at $220 \mathrm{~K}$ the narrow Lorentzian-shaped component shows a line width of approximately $1.9 \mathrm{kHz}$ while the broad one is characterized by $\delta=8.7 \mathrm{kHz}$. The area fraction $A_{\mathrm{f}}$ of the narrow line, which reflects the number fraction of fast $\mathrm{Li}$ ions with jump rates larger than about $10^{4} \mathrm{~s}^{-1}$, amounts to approximately $19 \%$ at this temperature. Values of $A_{\mathrm{f}}$ have been estimated by fitting the spectra with a combination of two Voigt functions. Interestingly, $A_{\mathrm{f}}$ steadily increases with increasing $T$ reaching $28 \%$ at $233 \mathrm{~K}, 35 \%$ at $243 \mathrm{~K}$, and $41 \%$ at $253 \mathrm{~K}$. At a temperature of $263 \mathrm{~K}$ nearly $50 \%$ of the total number of Li ions participate in a very fast $\mathrm{Li}$ diffusion process. The corresponding line width of the sharp component became smaller than $1 \mathrm{kHz}$. Thus, with rising temperature the fractional amount of $\mathrm{Li}$ ions being highly mobile on the NMR time scale increase in an almost linear fashion (Fig. 13c), a similar behaviour has been found for nanocrystalline $\mathrm{LiNbO}_{3} \cdot{ }^{38}$ Let us note that at higher temperatures a separation of the two spin reservoirs with their distinct dynamics turns out to be difficult. This is due to the fact that the broad spectral component, representing rather
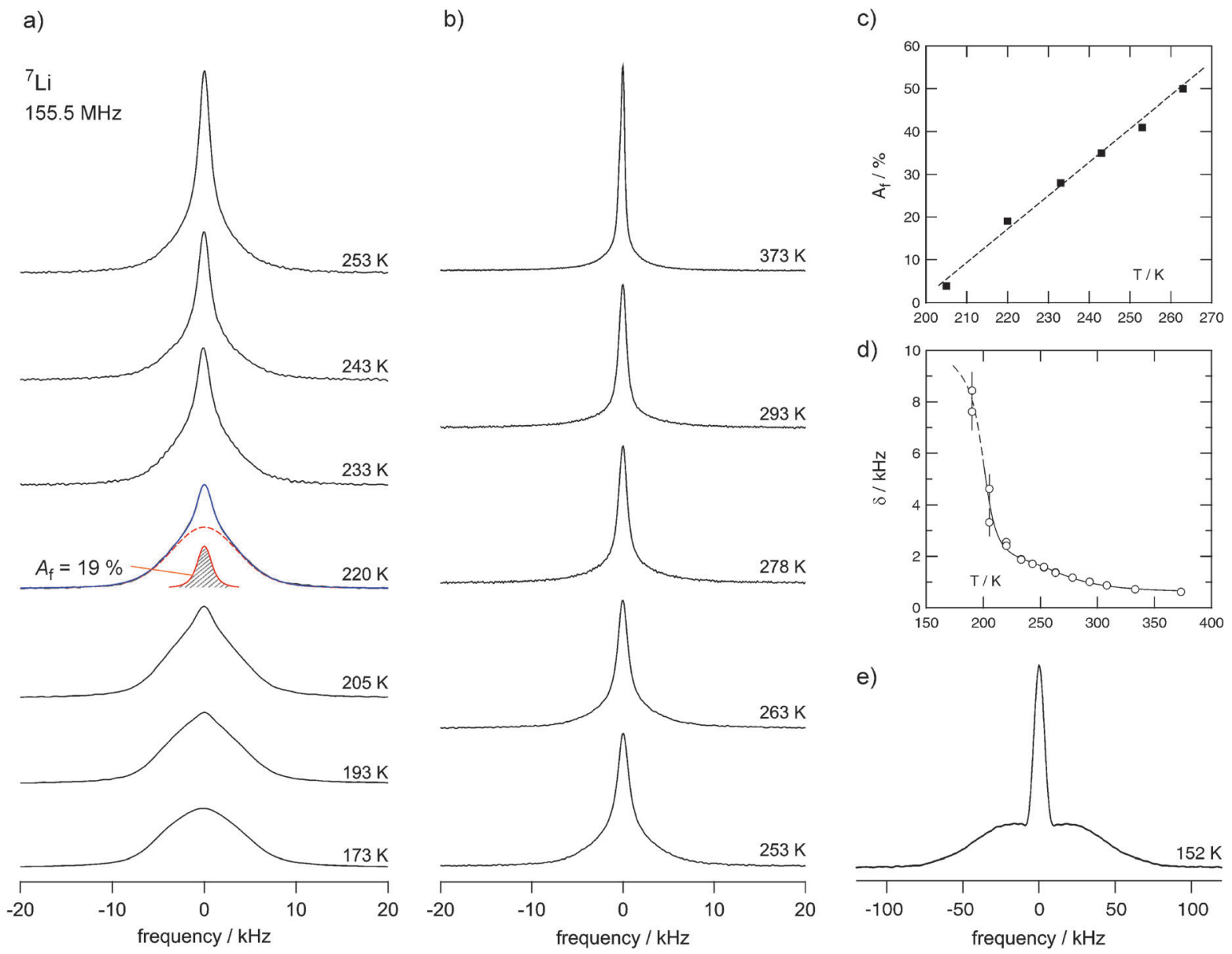

Fig. 13 (a) and (b) ${ }^{7} \mathrm{Li}$ NMR spectra of a non-rotating sample of cubic LLZO $(0.9 \mathrm{wt} \% \mathrm{Al})$ recorded at $155.5 \mathrm{MHz}$ in the temperature range from $173 \mathrm{~K}$ to $373 \mathrm{~K}$. As an example, the two-component NMR line at $220 \mathrm{~K}$ is represented by a superposition of two Voigt functions, that is, a combination of a Gaussian and a Lorentzian. The area fraction $A_{\mathrm{f}}=f(T)$ of the narrow contribution (solid line), representing very fast Li ions in LLZO, amounts to approximately $19 \%$. The dashed line shows the dipolarly broadened component which reflects slow Li ions. See text for further details. (c) Temperature dependence of $A_{\mathrm{f}}$, (d) NMR line width $d$ of the narrow component highlighted in (a), (e) ${ }^{7} \mathrm{Li}$ stimulated echo NMR spectrum recorded at $152 \mathrm{~K}$ in order to reveal quadrupole intensities next to the Gaussian-shaped central transition. 
slow Li ions in LLZO, steadily decreases in intensity. The heterogeneous $\mathrm{MN}$ observed at low $T$ might reflect a large distribution of Li jump rates. Such a distribution is expected to be preserved even at temperatures larger than $300 \mathrm{~K}$. Interestingly, even the line width of the sharp component reveals a stepwise narrowing reflecting a transition from partial to full averaging of dipolar interactions. Starting from $2 \mathrm{kHz}$ at $263 \mathrm{~K}$ the line width is further decreased with increasing $T$ finally reaching $800 \mathrm{~Hz}$ in the regime of extreme narrowing (see Fig. 13d). This is very similar to the behaviour observed by ${ }^{7} \mathrm{Li}$ NMR spin-spin-relaxation measurements discussed below (see Fig. 14).

In addition to NMR line shapes, ${ }^{7} \mathrm{Li}$ NMR spin-lattice relaxation (SLR) rates have been recorded in both, the laboratory and rotating frames of reference, see ref. 21, 37, and 39 for an introduction into these NMR techniques. It is worth mentioning that the two components of the NMR line are characterized by the same ${ }^{7}$ Li SLR NMR rates $R_{1}$ and $R_{1 \rho}$. Thus, a separation of the two, dynamically different spin reservoirs according to that shown previously for the nanocrystalline two-phase $\mathrm{Li}$ conductor $\mathrm{Li}_{2} \mathrm{O}: \mathrm{Al}_{2} \mathrm{O}_{3},{ }^{35,40}$ is not possible. In contrast to the present work, in the previous study two spin ensembles, which seem to be unaffected by spin-diffusion effects, were anticipated to be also spatially separated. $^{29}$ The temperature dependence of the rates of the sintered sample of cubic LLZO, which have been recorded at different frequencies, are shown in Fig. 14 in an Arrhenius plot. Below $180 \mathrm{~K}$, i.e., in the rigid-lattice regime (see above), the rates are governed by non-diffusive background relaxation and reveal a weaker-than-activated temperature dependence. Presumably, the SLR NMR rates in this temperature range are influenced by lattice vibrations, interactions between the spins and paramagnetic impurities and/or by strictly localized, i.e., caged ion dynamics which do not reflect translational jump processes. However, at higher temperatures the SLR

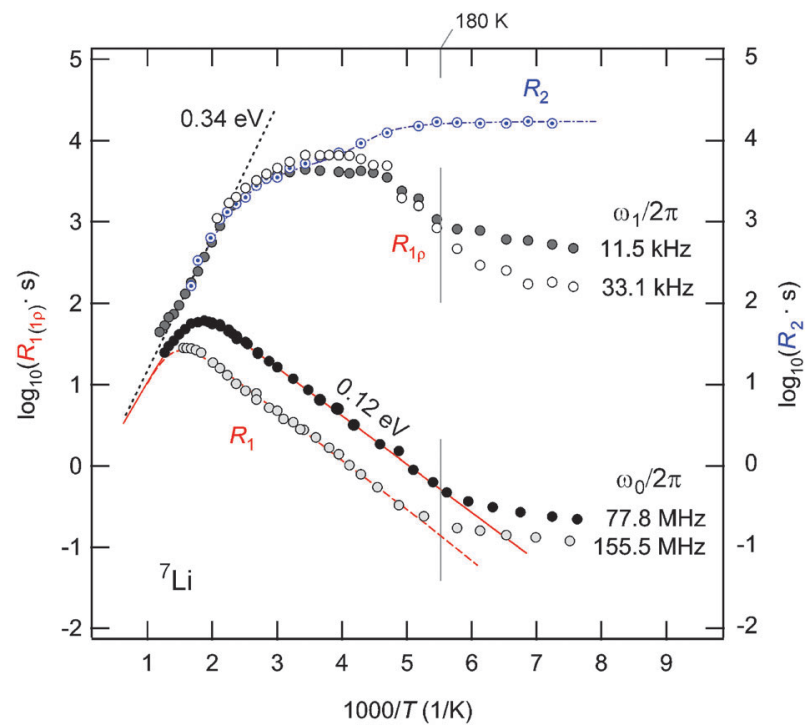

Fig. 14 Temperature dependence of the ${ }^{7} \mathrm{Li}$ NMR spin-spin $\left(R_{2}\right)$ as well as spin-lattice relaxation rates $\left(R_{1}\right.$ and $\left.R_{1 \rho}\right)$ of cubic LLZO. The NMR spin-lattice relaxation rates were measured at the frequencies indicated. Spin-spin relaxation rates were recorded at a resonance frequency of $77.8 \mathrm{MHz}$. See text for further details.
NMR rates pass through characteristic diffusion-induced rate peaks from which Li jump rates $1 / \tau$ and activation energies $E_{\mathrm{a}, \mathrm{NMR}}$ can be deduced. Interestingly, the rates recorded at $77.8 \mathrm{MHz}$ reveal an asymmetric diffusion-induced peak $R_{1}(1 / T)$. In general, the low-temperature flank, for which $\omega_{0} \tau \gg 1$ holds, is influenced by short-range Li motions which might be affected by correlation effects (see below). ${ }^{21,37,39,40}$ Compared with this, on the high-temperature side of the rate peak, i.e., in the limit $\omega_{0} \tau \ll 1$, the SLR rate is governed by long-range Li transport. ${ }^{21,37,39,40}$

The mean diffusion parameters characterizing the latter are directly comparable with the results obtained from dc conductivity measurements (see above). The solid line in Fig. 14 represents a fit according to the model introduced by Bloembergen, Purcell and Pound ${ }^{41}$ which was originally developed for isotropic uncorrelated diffusion: $R_{1} \propto \tau /[1+$ $\left.\left.\omega_{0} \tau\right)^{\beta}\right]$. Here, the asymmetry of the rate peak is taken into account by the parameter $\beta$, which can adopt values ranging from 1 to 2 . $\beta=2$, the so-called BPP-behaviour, is obtained for uncorrelated motion. $\beta<2$ indicates that the underlying hopping correlation function $G(t)$ is represented by a nonexponential rather than an exponential function. Correlation effects such as structural disorder and/or Coulomb interactions are considered to be responsible for the deviation from exponential time behaviour of $G(t)$. In the present case, $\beta$ amounts to about 1.4 ; the activation energy $E_{\mathrm{a}, \mathrm{NMR}}$ turns out to be approximately $0.31 \mathrm{eV}$. This value is in good agreement with that probed by impedance spectroscopy (see above). For comparison with $E_{\mathrm{a}, \mathrm{NMR}}$, the corresponding activation energy in the limit $\omega_{0} \tau \gg 1$ turns out to be about $E_{\mathrm{a}, \mathrm{NMR}}^{\prime}=0.12 \mathrm{eV}$. In general, $E_{\mathrm{a}, \mathrm{NMR}}^{\prime}$ and $E_{\mathrm{a}, \mathrm{NMR}}$ are linked with each other via $E_{\mathrm{a}, \mathrm{NMR}}^{\prime}=(\beta-1) E_{\mathrm{a}, \mathrm{NMR}} \cdot{ }^{37}$ In addition, $\beta$ determines the frequency dependence of $R_{1}$ in the limit $\omega_{0} \tau \gg 1$ according to $R_{1} \propto \omega_{0}^{-\beta}$. No frequency dependence is expected in the limit $\omega_{0} \tau \ll 1$ when $3 \mathrm{D}$ diffusion is regarded. ${ }^{37}$ This is in contrast to ion conductors showing low-dimensional Li diffusion. ${ }^{37,42-44}$

Owing to technical limitations of our NMR setup, temperatures higher than $800 \mathrm{~K}$ could not be reached. Thus, the hightemperature flank of the $R_{1}$ rate peak recorded at $77.8 \mathrm{MHz}$ was only partly accessible. However, with the help of SLR NMR measurements performed in the rotating frame of reference and carried out at locking frequencies $\omega_{1} / 2 \pi$ in the $\mathrm{kHz}$ the complete high-temperature flank of the corresponding diffusion-induced rate peak can be probed. The dotted line in Fig. 14 represents an Arrhenius fit of some of the data points of the high- $T$ flank of the $R_{1 \rho}$ data recorded at $\omega_{1} / 2 \pi=$ $11.5 \mathrm{kHz}$. The fit yields an activation energy of approximately $0.34 \mathrm{eV}$ and is in good agreement with the trend of the ${ }^{7} \mathrm{Li}$ NMR spin-spin relaxation rates $R_{2}$ which are also included in Fig. 13. The $R_{1 \rho}$ measurements are limited by $R_{2}$ leading to an apparently broad rate peak whose top is cut off. Nevertheless the temperature at which the rate maximum appears can be estimated to be approximately $300 \mathrm{~K}$. In general, the diffusioninduced SLR rate maximum shows up when the Li correlation rate $1 / \tau^{\prime}$ reaches the order of the angular frequencies $\omega_{0}$ and $\omega_{1}$, respectively. ${ }^{37} 1 / \tau^{\prime}$ is expected to be identical with the mean jump rate $1 / \tau$ within a factor of two. In Fig. 15 the Li 
jump rates $1 / \tau_{\mathrm{NMR}}$ directly deduced from the rate maxima according to $\omega_{0} \tau_{\mathrm{NMR}}=1$ and $\omega_{1} \tau_{\mathrm{NMR}}=0.5$, see ref. 39, respectively, are shown in an Arrhenius plot. The additional vertical axis in Fig. 15 converts the rates $1 / \tau_{\mathrm{NMR}}$ into selfdiffusion coefficients according to the Einstein-Smoluchowski equation: $D_{\mathrm{NMR}}=a_{\mathrm{j}}^{2} /\left(6 \tau_{\mathrm{NMR}}\right)$. The mean jump distance $a_{\mathrm{j}}$ was estimated to be approximately $0.2 \mathrm{~nm}$. In analogy to previous studies ${ }^{21,45,46}$ comparing results obtained from NMR with those from impedance spectroscopy, dc conductivity values $\sigma_{\mathrm{DC}}$ (Fig. 11) have been roughly converted into $\mathrm{Li}$ jump rates using both the Nernst-Einstein and EinsteinSmoluchowski equations. As is well-known, the combination of the two relations yields

$$
\tau_{\sigma}^{-1}=H_{\mathrm{R}} f_{\mathrm{c}} \frac{6 k_{\mathrm{B}} T}{N q^{2} a_{\mathrm{j}}^{2}} \sigma_{\mathrm{DC}}
$$

where $N$ denotes the number density of charge carriers and $q$ the charge of the Li ions. The product of Haven ratio $H_{\mathrm{R}}$ and correlation factor $f_{\mathrm{c}}$ was assumed to be 1 . Additionally, in Fig. 15 electrical relaxation rates $1 / \tau_{M}$ are included which were obtained from the imaginary part of the electrical modulus $M^{\prime \prime}$ which has been analyzed as a function of frequency $\nu$. Modulus spectra $M^{\prime \prime}(\nu)$ were recorded at various temperatures $T$ (not shown here for the sake of brevity). The characteristic maxima show up when $1 / \tau_{M^{\prime \prime}}$ is of the order of the angular frequency $\nu 2 \pi$. The solid line in Fig. 15 represents a fit taking into account $1 / \tau_{M^{\prime \prime}}$ and $1 / \tau_{\sigma}$ yielding $0.34 \mathrm{eV}$ (see above).

The jump rates $1 / \tau_{\mathrm{NMR}}$ obtained from NMR $R_{1}$ measurements are in fair agreement with the conductivity results. The agreement between $1 / \tau_{\sigma}$ and $1 / \tau_{\mathrm{NMR}}$ probed via $R_{1 \rho}$ turns out to be somewhat better which might be due to the fact that the two methods probe $\mathrm{Li}$ dynamics on a very similar time scale. For the sake of completeness, a Li jump rate is included in Fig. 15 which has been estimated from NMR line narrowing (Fig. 8). Slightly above $200 \mathrm{~K}$ the narrow

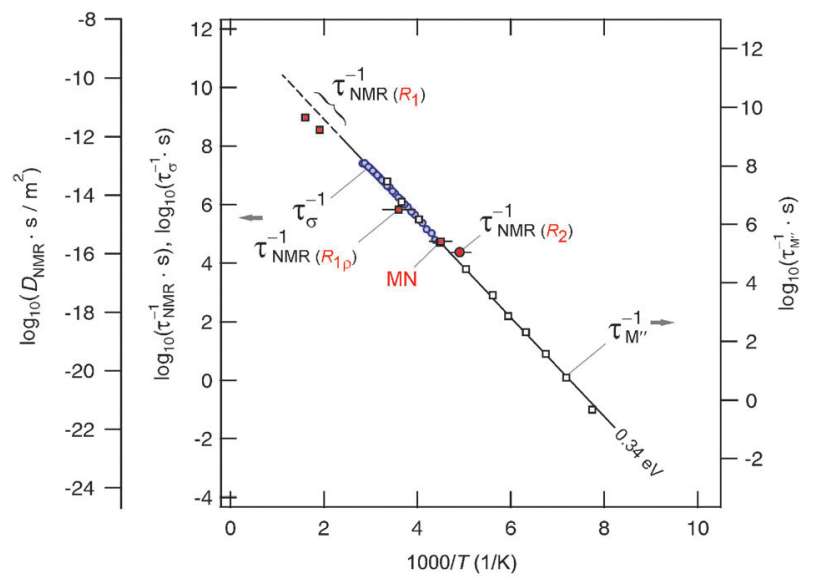

Fig. 15 Temperature dependence of the Li jump rates of cubic LLZO $(0.9 \mathrm{wt} \% \mathrm{Al})$ obtained from NMR $1 / \tau_{\mathrm{NMR}}$ and impedance spectroscopy $1 / \tau_{\mathrm{s}}$. For comparison, electrical relaxation rates $1 / \tau_{M^{\prime \prime}}$ deduced from the peaks of various modulus spectra $M^{\prime \prime}(\nu)$ are also included. The solid line shows an Arrhenius fit yielding $0.34 \mathrm{eV}$. The jump rate marked with MN denotes a value deduced from NMR line narrowing. See text for further details. component shows up. At $225 \mathrm{~K}$ the corresponding line width is reduced to $\delta_{\mathrm{rl}} / 2$ and the associated jump rate is given by $1 / \tau_{\mathrm{NMR}}=\delta_{\mathrm{r} 1} 2 \pi$. For comparison, at $205 \mathrm{~K}$ the rates $R_{2}$ start to deviate from their rigid-lattice value $R_{20}=60 \mu$ s leading to $1 / \tau_{\mathrm{NMR}} \approx R_{20}$ at this temperature. This jump rate is also included in Fig. 15. Taken together, the jump rates probed by NMR spectroscopy corroborate the transport parameters of a sintered sample of cubic LLZO obtained from impedance spectroscopy. In agreement with line widths measurements (Fig. 13d), $R_{2}$ shows a two-step decrease with increasing temperature indicating that the $\mathrm{Li}$ ions get access to further diffusion pathways at elevated temperatures.

\section{Conclusions}

We have synthesized the two different structural modifications of LLZO and found a certain Al content to be necessary for the formation of cubic LLZO. One already could speculate from the patent by $\mathrm{Yoshida}^{47}$ that $\mathrm{Al}$ is a necessary ingredient for the formation of cubic LLZO. As in the present work, the recently published papers ${ }^{10-13}$ state that cubic LLZO only forms when $\mathrm{Al}$ is present during synthesis but there was no definite knowledge about the role of Al. With the combination of XRD, TEM-PED and neutron scattering we are able to propose a structure model which takes both $\mathrm{Al}$ and $\mathrm{Li}$ ions into account. Neutron diffraction measurements show a high degree of $\mathrm{Li}$ disorder even at $4 \mathrm{~K}$ which becomes even more pronounced at $300 \mathrm{~K}$. We regard this disorder as the reason for the high mobility of the Li ions in the cubic garnet-type lattice. As proved by ${ }^{27}$ Al MAS NMR spectroscopy most of the Al ions are located on the $24 d$ site. With the results of phase transformation during synthesis we regard the $\mathrm{Al}$ ions on the $24 d$ site to stabilize the cubic garnet structure.

NMR was found to be a very sensitive probe for small structural changes in the garnet-type lattice. NMR shows that minor changes of the Al distribution during the sintering process cause the Li disorder to become even more pronounced than in the powder before sintering. The TEM-PED patterns agree very well with our proposed structure model and the corresponding pattern simulation; other structure models fit less well. The simulated structure image also fits well with the HRTEM image, confirming our cubic structure model.

Electrochemical measurements performed with different LLZO samples confirm that the conductivity depends on the Al content, i.e., the degree of transformation from the tetragonal to the cubic crystal structure. Ac and dc conductivities show almost identical values approving the high conductivity also under current load. Li jump rates deduced from NMR spin-lattice relaxation measurements agree well with those calculated from electrical conductivity data. Moreover, the activation energies determined by both techniques agree very well. The electronic conductivity and the corresponding electronic transference number are very small, and LLZO was found to be stable against reaction with $\mathrm{Li}$ metal over the measured period of several days. Therefore, we suppose that Al-stabilized cubic LLZO is a suitable Li solid electrolyte. Tests of the compatibility of LLZO with various cathode materials and measurements of the overvoltages occurring 
in contact with these as well as Li metal under current load are in progress and will help further clarify the applicability of cubic LLZO as a solid state electrolyte for Li ion batteries.

\section{Acknowledgements}

We acknowledge financial support by the German Research Foundation (DFG) within the project "High performance lithium batteries" (DFG JA 648/15-1 and 15-2). We also acknowledge helpful discussion within the BASF research network for Batteries and Electrochemistry. Furthermore financial support by the Federal Ministry of Education and Research (BMBF) within the projects "Competence in Electrochemistry for Electromobility" and "Lithium Ion Batteries LIB 2015" both for the Gießen and the Hannover teams is gratefully acknowledged.

The authors benefitted from discussions with Alan Logéat, Thomas Köhler and Ulrich Eisele from Robert Bosch GmbH and thank Christoph Essig for the ICP-OES analysis of the garnet samples.

\section{Notes and references}

1 K. Xu, Chem. Rev., 2004, 104, 4303-4417.

2 D. Aurbach, J. Power Sources, 2000, 89, 206-218.

3 D. Aurbach, B. Markovsky, G. Salitra, E. Markevich, Y. Talyossef, M. Koltypin, L. Nazar, B. Ellis and D. Kovacheva, J. Power Sources, 2007, 165, 491-499.

4 J. Vetter, P. Novak, M. R. Wagner, C. Veit, K. C. Moller, J. O. Besenhard, M. Winter, M. Wohlfahrt-Mehrens, C. Vogler and A. Hammouche, J. Power Sources, 2005, 147, 269-281.

5 V. Thangadurai, H. Kaack and W. J. F. Weppner, J. Am. Ceram. Soc., 2003, 86, 437-440.

6 R. Murugan, V. Thangadurai and W. Weppner, Angew. Chem., Int. Ed., 2007, 46, 7778-7781.

7 J. Awaka, N. Kijima, H. Hayakawa and J. Akimoto, J. Solid State Chem., 2009, 182, 2046-2052.

8 J. Awaka, A. Takashima, K. Kataoka, N. Kijima, Y. Idemoto and J. Akimoto, Chem. Lett., 2011, 60-62.

9 K. H. Kim, Y. Iriyama, K. Yamamoto, S. Kumazaki, T. Asaka, K. Tanabe, C. A. J. Fisher, T. Hirayama, R. Murugan and Z. Ogumi, J. Power Sources, 2011, 196, 764-767.

10 C. A. Geiger, E. Alekseev, B. Lazic, M. Fisch, T. Armbruster, R. Langner, M. Fechtelkord, N. Kim, T. Pettke and W. Weppner, Inorg. Chem., 2011, 50, 1089-1097.

11 S. Kumazaki, Y. Iriyama, K.-H. Kim, R. Murugan, K. Tanabe, K. Yamamoto, T. Hirayama and Z. Ogumi, Electrochem. Commun., 2011, 13, 509-512.

12 Y. Jin and P. J. McGinn, J. Power Sources, 2011, DOI: 10.1016/ j.jpowsour.2011.05.065.

13 M. Kotobuki, K. Kanamura, Y. Sato and T. Yoshida, J. Power Sources, 2011, 196, 7750-7754.

14 M. Hoelzel, A. Senyshyn, R. Gilles, H. Boysen and H. Fuess, Neutron News, 2008, 18.
15 J. Rodriguez-Carvajal, Commission on Powder Diffraction Newsletter, 2001, 26, 12-19.

16 F. Izumi and R. A. Dilanian, Recent Research Developments in Physics, Transworld Research Network, 2002.

17 P. A. Stadelmann, Ultramicroscopy, 1987, 21, 131-145.

18 M. H. Hebb, J. Chem. Phys., 1952, 20, 185-190.

19 C. Wagner, J. Chem. Phys., 1953, 21, 1819-1827.

20 A. Kuhn, A. Düvel and P. Heitjans, 2011, unpublished results.

21 A. Kuhn, S. Narayanan, L. Spencer, G. Goward, V. Thangadurai and M. Wilkening, Phys. Rev. B: Condens. Matter Mater. Phys., 2011, 83, 094302.

22 E. J. Cussen, T. W. S. Yip, G. O'Neill and M. P. O'Callaghan, J. Solid State Chem., 2011, 184, 470-475.

23 L. Thieblot, J. Roux and P. Richet, Eur. J. Mineral., 1998, 10, $7-15$.

24 A. Grzechnik, H. Kruger, V. Kahlenberg and K. Friese, J. Phys.: Condens. Matter, 2006, 18, 8925-8934.

25 K. Momma and F. Izumi, J. Appl. Crystallogr., 2008, 41, 653-658.

26 F. Izumi, Solid State Ionics, 2004, 172, 1-6.

27 A. A. Belik, F. Izumi, T. Ikeda, V. A. Morozov, R. A. Dilanian, S. Torii, E. M. Kopnin, O. I. Lebedev, G. Van Tendeloo and B. I. Lazoryak, Chem. Mater., 2002, 14, 4464-4472.

28 K. Oikawa, T. Kamiyama, R. Kanno, F. Izumi, T. Ikeda and B. C. Chakoumakos, Mater. Sci. Forum, 2004, 443-444, 337.

29 K. Takada, H. Sakurai, E. Takayama-Muromachi, F. Izumi, R. A. Dilanian and T. Sasaki, Nature, 2003, 422, 53-55.

30 M. Yashima, J. Ceram. Soc. Jpn., 2009, 117, 1055-1059.

31 D. Jacob and P. Cordier, Ultramicroscopy, 2010, 110, 1166-1177.

32 L. Kienle, V. Duppel, B. Mogwitz, J. Janek, M. v. Kreutzbruck, A. Leineweber and A. Simon, Cryst. Growth Des., 2011, 11, 2412-2421.

33 I. M. Hodge, M. D. Ingram and A. R. West, J. Electroanal. Chem., 1976, 74, 125-143.

34 A. D. Pelton, Bull. Alloy Phase Diagrams, 1986, 7, 228-231.

35 M. Wilkening, S. Indris and P. Heitjans, Phys. Chem. Chem. Phys., 2003, 5, 2225-2231.

36 B. Koch and M. Vogel, Solid State Nucl. Magn. Reson., 2008, 34, $37-43$.

37 P. Heitjans and J. Kärger, Diffusion in Condensed MatterMethods, Materials, Models, Springer, Berlin, 2005.

38 P. Heitjans, M. Masoud, A. Feldhoff and M. Wilkening, Faraday Discuss., 2007, 134, 67-82.

39 W. Bensch, T. Bredow, H. Ebert, P. Heitjans, S. Indris, S. Mankovsky and M. Wilkening, Prog. Solid State Chem., 2009, 37, 206-225.

40 M. Wilkening and P. Heitjans, Phys. Rev. B: Condens. Matter Mater. Phys., 2008, 77, 024311.

41 N. Bloembergen, E. M. Purcell and R. V. Pound, Phys. Rev., 1948, 73, 679-712.

42 V. Epp and M. Wilkening, Phys. Rev. B: Condens. Matter Mater. Phys., 2010, 82, 020301.

43 W. Küchler, P. Heitjans, A. Payer and R. Schöllhorn, Solid State Ionics, 1994, 70, 434-438.

44 A. Kuhn, P. Sreeraj, R. Pöttgen, H. D. Wiemhofer, M. Wilkening and P. Heitjans, J. Am. Chem. Soc., 2011, 133, 11018.

45 M. Wilkening, C. Mühle, M. Jansen and P. Heitjans, J. Phys. Chem. B, 2007, 111, 8691-8694.

46 M. Wilkening, A. Kuhn and P. Heitjans, Phys. Rev. B: Condens. Matter Mater. Phys., 2008, 78, 054303.

47 United States Pat., US 2010/0047696 A1, 2010. 